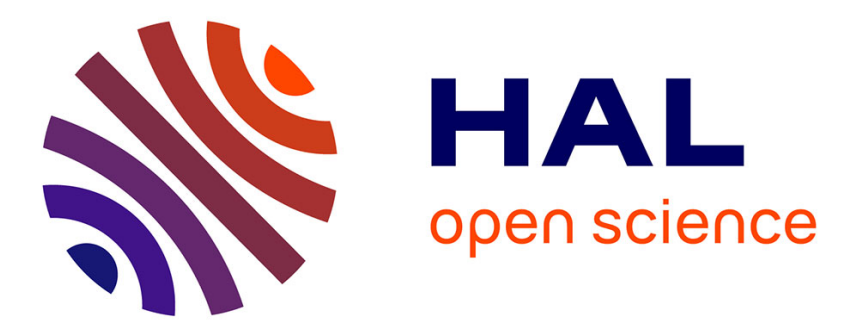

\title{
Le faisceau occipito-frontal inférieur sous-tend la voie ventrale sémantique directe du langage: une approche multimodale
}

Fabien Almairac

\section{To cite this version:}

Fabien Almairac. Le faisceau occipito-frontal inférieur sous-tend la voie ventrale sémantique directe du langage: une approche multimodale. Médecine humaine et pathologie. 2013. dumas-01010690

\section{HAL Id: dumas-01010690 https://dumas.ccsd.cnrs.fr/dumas-01010690}

Submitted on 20 Jun 2014

HAL is a multi-disciplinary open access archive for the deposit and dissemination of scientific research documents, whether they are published or not. The documents may come from teaching and research institutions in France or abroad, or from public or private research centers.
L'archive ouverte pluridisciplinaire $\mathbf{H A L}$, est destinée au dépôt et à la diffusion de documents scientifiques de niveau recherche, publiés ou non, émanant des établissements d'enseignement et de recherche français ou étrangers, des laboratoires publics ou privés. 
UNIVERSITE DE NICE SOPHIA-ANTIPOLIS

FACULTE DE MEDECINE DE NICE

Année 2013

THESE POUR LE DIPLOME D'ETAT DE DOCTEUR EN MEDECINE

Le faisceau occipito-frontal inferieur sous-tend la voie ventrale sémantique directe du langage : une approche multimodale

Présentée et soutenue publiquement le 25 juin 2013

Par M. Fabien ALMAIRAC

Né le 10 février 1983 à Grasse (06)

\section{JURY}

Président : $\quad$ Monsieur le Professeur Philippe PAQUIS

Assesseurs : $\quad$ Monsieur le Docteur Denys FONTAINE

Madame le Docteur Christine LEBRUN-FRENAY

Monsieur le Professeur Michel LONJON

Directeur de Thèse : Monsieur le Professeur Hugues DUFFAU 


\section{RESUME}

Introduction : Les modèles neuropsychologiques et anatomo-fonctionnels récents du langage suggèrent une organisation selon une double voie parallèle et synchrone. La voie dorsale, sous-tendue par le faisceau arqué (FA), est impliquée dans les processus phonologiques. La voie ventrale est impliquée dans les traitements sémantiques du langage, mais la connectivité qui la sous-tend est discutée.

Objectifs : Démontrer l'implication du faisceau fronto-occipital inférieur (FOFI) dans le traitement sémantique du langage par une approche multi-modale.

Méthodes: Des malades opérés en condition éveillée d'un gliome de bas grade hémisphérique gauche ont eu une évaluation de la fluence verbale sémantique et phonologique, en pré-opératoire, post-opératoire immédiat et à trois mois. Les structures neurales impliquées dans le langage ont été identifiées et préservées grâce à une cartographie corticale et sous-corticale par stimulation électrique directe (SED). Les données orthophoniques ont été confrontées à une analyse voxel par voxel afin d'établir des cartographies d'association statistiques anatomo-fonctionnelles. Des tests de corrélation et des superpositions ont été faits entre les voxels infiltrés et les faisceaux d'association de l'atlas radiologique de Catani.

Résultats : En pré-opératoire, les voxels infiltrés de la substance blanche ventro-latérale étaient significativement associés aux scores de fluence sémantique $(\mathrm{p}<0,05)$. Ces voxels étaient superposables à $37,7 \%$ à ceux du FOFI de l'atlas radiologique. Une corrélation négative entre les scores de fluence sémantique et le nombre de voxels infiltrés du FOFI était mise en évidence $(r=-0,4 ; \mathrm{p}=0,029)$. Il n'y avait pas de corrélation ni d'association avec les scores phonologiques ou avec ceux impliquant le FA. En post-opératoire, les scores de fluence (sémantique et phonologique) à 3 mois étaient identiques à ceux obtenus en préopératoire, avec un déficit transitoire constaté lors de l'évaluation post-opératoire immédiate. Il n'y avait pas de relation statistique entre les cavités de résection et les scores de fluence verbale.

Conclusion: Le FOFI semble avoir une implication spécifique et nécessaire dans les processus sémantiques du langage. Ce faisceau s'intègre dans l'organisation hodotopique du langage (sous-réseaux interagissant ensemble), dont la préservation au cours de la chirurgie est indispensable au maintien de la fonction.

Mots-clés : faisceau fronto-occipital inférieur; processus sémantiques du langage; stimulation électrique directe; cartographie anatomo-fonctionnelle; gliome diffus de bas grade 


\section{Summary}

Background: Contemporary brain models of language suggest a dual-route model. The dorsal stream is involved in phonological processing and is underlied by the arcuate fascicle (AF). The ventral stream is involved in semantic processing but there is a great deal of controversy regarding its connectivity.

Objectives: To give further neuropsychological-based evidence for the involvement of the left inferior fronto-occipital fascicle (IFOF) in semantic processing by a multilevel approach.

Methods: To this end, we assessed phonological and semantic language processing with a prototypic task of language (i.e. verbal fluency) in a homogeneous group of 31 patients with a left diffuse low-grade glioma just before, immediately after and three months after the brain surgery. All were operated on under local anesthesia with cortical and subcortical direct electro-stimulation in order to identify and preserve eloquent brain structures for language during tumor resection. Behavioral data were confronted to conventional voxelwise-based analyses. Correlations and overlapping were also conducted from a diffusion tensor imaging fascicle atlas.

Results: Preoperatively, we found a significant association between semantic fluency scores and classical language areas including inferior frontal gyrus and temporal regions but also in the ventro-lateral white matter $(P<0.05$ corrected). The statistical map was found to broadly overlap with the spatial position of the left IFOF (37.7\%). Further analyses demonstrated that the degree of left IFOF infiltration was related to decreased performance $(r=-0.4, P=0.029)$. Following the same analyses, no significant result was demonstrated for the phonological task and for analyses involving the AF. Postoperatively, verbal fluency Z-scores transiently decreased in the immediate postoperative period, but returned to baseline preoperative scores three months after surgery. Voxelwise analyses conducted on resective cavity maps did not reveal any significant behavior/lesion relationship and that for both tasks.

Conclusion: These results strengthen the brain hodotopical organization of language networks (i.e. large-scale sub-networks interacting together), thanks to the preservation of cortico-subcortical structures mandatory for language function during surgery. This overall pattern of results provides neuropsychological evidence for an essential role of the left IFOF in semantic processing.

Keywords: inferior fronto-occipital fascicle; language semantics; direct electrical stimulation; voxelwise lesion-behaviour mapping; low-grade glioma 


\section{FACULTE DE MEDECINE}

Liste des professeurs au 1er mars 2013 à la Faculté de Médecine de Nice

Doyen

Assesseurs

Conservateur de la bibliothèque

Chef des services administratifs

Doyens Honoraires

\section{Professeurs Honoraires}

\author{
M. BALAS Daniel \\ M. BLAIVE Bruno \\ M. BOQUET Patrice \\ M. BOURGEON André \\ M. BRUNETON Jean-Noël \\ Mme BUSSIERE Françoise \\ M. CHATEL Marcel \\ M. COUSSEMENT Alain \\ M. DARCOURT Guy \\ M. DELMONT Jean \\ M. DEMARD François \\ M. DOLISI Claude \\ M. FREYCHET Pierre \\ M. GILLET Jean-Yves \\ M. GRELLIER Patrick \\ M. HARTER Michel \\ M. INGLESAKIS Jean-André
}

M.C.A. Honoraire

M.C.U. Honoraires
M. BAQUÉ Patrick

M. BOILEAU Pascal

M. HEBUTERNE Xavier

M. LEVRAUT Jacques

M. SCALABRE Grégory

Mme HIZEBRY Valérie

M. AYRAUD Noël

M. RAMPAL Patrick

M. LALANNE Claude-Michel M. LAMBERT Jean-Claude

M. LAPALUS Philippe M. LAZDUNSKI Michel M. LEFEBVRE Jean-Claude M. LE BAS Pierre M. LE FICHOUX Yves M. LOUBIERE Robert M. MARIANI Roger M. MASSEYEFF René M. MATTEI Mathieu M. MOUIEL Jean Mme MYQUEL Martine M. OLLIER Amédée M. SCHNEIDER Maurice M. SERRES Jean-Jacques M. TOUBOL Jacques M. TRAN Dinh Khiem M. ZIEGLER Gérard

Mlle ALLINE Madeleine

M. ARNOLD Jacques M. BASTERIS Bernard Mlle CHICHMANIAN Rose-Marie M. EMILIOZZI Roméo M. GASTAUD Marcel M. GIRARD-PIPAU Fernand Mme MEMRAN Nadine M. MENGUAL Raymond M. POIREE Jean-Claude Mme ROURE Marie-Claire 


\section{PROFESSEURS CLASSE EXCEPTIONNELLE}

M. BENCHIMOL Daniel

M. CAMOUS Jean-Pierre

M. DELLAMONICA Pierre

M. DESNUELLE Claude

Mme EULLER-ZIEGLER Liana

M. FENICHEL Patrick

M. FUZIBET Jean-Gabriel

M. FRANCO Alain

M. GASTAUD Pierre

M. GERARD Jean-Pierre

M. GILSON Éric

M. GRIMAUD Dominique

M. HEBUTERNE Xavier

M. HOFMAN Paul

M. LACOUR Jean-Philippe

Mme LEBRETON Elisabeth

M. ORTONNE Jean-Paul

M. PRINGUEY Dominique

M. SANTINI Joseph

M. THYSS Antoine

M. VAN OBBERGHEN Emmanuel
Chirurgie Générale (53.02)

Thérapeutique (48.04)

Maladies Infectieuses ; Maladies Tropicales (45.03)

Biologie Cellulaire (44.03)

Rhumatologie (50.01)

Biologie du Développement et de la Reproduction (54.05)

Médecine Interne (53.01)

Gériatrie et Biologie du vieillissement (53-01)

Ophtalmologie (55.02)

Cancérologie ; Radiothérapie (47.02)

Biologie Cellulaire (44.03)

Anesthésiologie et Réanimation Chirurgicale (48.01)

Nutrition (44.04)

Anatomie et Cytologie Pathologiques (42.03)

Dermato-Vénéréologie (50.03)

Chirurgie Plastique, Reconstructrice et Esthétique (50.04)

Dermato-Vénéréologie (50.03)

Psychiatrie d'Adultes (49.03)

O.R.L. (55.01)

Cancérologie ; Radiothérapie (47.02)

Biochimie et Biologie Moléculaire (44.01)

\section{PROFESSEURS PREMIERE CLASSE}

M. AMIEL Jean

M. BATT Michel

M. BERARD Etienne

M. BERNARDIN Gilles

M. BOILEAU Pascal

M. BONGAIN André

Mme CRENESSE Dominique

M. DARCOURT Jacques

M. DE PERETTI Fernand

M. DRICI Milou-Daniel

M. ESNAULT Vincent

M. GIBELIN Pierre

M. GUGENHEIM Jean

M. HASSEN KHODJA Reda

Mme ICHAI Carole

M. LONJON Michel

M. MARQUETTE Charles-Hugo

M. MARTY Pierre

M. MICHIELS Jean-François

M. MOUNIER Nicolas

M. MOUROUX Jérôme

M. PADOVANI Bernard

M. PAQUIS Philippe

Mme PAQUIS Véronique

M. QUATREHOMME Gérald

M. RAUCOULES-AIME Marc

Mme RAYNAUD Dominique

M. ROBERT Philippe

M. ROSENTHAL Eric
Urologie (52.04)

Chirurgie Vasculaire (51.04)

Pédiatrie (54.01)

Réanimation Médicale (48.02)

Chirurgie Orthopédique et Traumatologique (50.02)

Gynécologie-Obstétrique (54.03)

Physiologie (44.02)

Biophysique et Médecine Nucléaire (43.01)

Anatomie-Chirurgie Orthopédique (42.01)

Pharmacologie Clinique (48.03)

Néphrologie (52-03)

Cardiologie (51.02)

Chirurgie Digestive (52.02)

Chirurgie Vasculaire (51.04)

Anesthésiologie et Réanimation Chirurgicale (48.01)

Neurochirurgie (49.02)

Pneumologie (51.01)

Parasitologie et Mycologie (45.02)

Anatomie et Cytologie Pathologiques (42.03)

Cancérologie ; Radiothérapie (47.02)

Chirurgie Thoracique et Cardio-Vasculaire (51.03)

Radiologie et Imagerie Médicale (43.02)

Neurochirurgie (49.02)

Génétique (47.04)

Médecine Légale et Droit de la Santé (46.03)

Anesthésie et Réanimation Chirurgicale (48.01)

Hématologie (47.01)

Psychiatrie d'Adultes (49.03)

Médecine Interne (53.01) 
M. SCHNEIDER Stéphane

M. TRAN Albert
Nutrition (44.04)

Hépato-Gastroentérologie (52.01)

PROFESSEURS DEUXIEME CLASSE

\begin{tabular}{|c|c|}
\hline M. & ALBERTINI Marc \\
\hline Mme & ASKENAZY-GITTARD Florence \\
\hline M. & BAHADORAN Philippe \\
\hline M. & BAQUE Patrick \\
\hline Mme & BLANC-PEDEUTOUR Florence \\
\hline M. & BOUTTE Patrick \\
\hline Mlle & BREUIL Véronique \\
\hline M. & CANIVET Bertrand \\
\hline M. & CARLES Michel \\
\hline M. & CASSUTO Jill-Patrice \\
\hline M. & CASTILLO Laurent \\
\hline M. & CHEVALLIER Patrick \\
\hline M. & DUMONTIER Christian \\
\hline M. & FERRARI Emile \\
\hline M. & FERRERO Jean-Marc \\
\hline M. & FOURNIER Jean-Paul \\
\hline M. & FREDENRICH Alexandre \\
\hline Mlle & GIORDANENGO Valérie \\
\hline M. & GUERIN Olivier \\
\hline M. & HANNOUN-LEVI Jean-Michel \\
\hline M. & JOURDAN Jacques \\
\hline M. & LEVRAUT Jacques \\
\hline M. & PASSERON Thierry \\
\hline M. & PRADIER Christian \\
\hline M. & ROGER Pierre-Marie \\
\hline M. & ROHRLICH Pierre \\
\hline M. & RUIMY Raymond \\
\hline M. & SADOUL Jean-Louis \\
\hline M. & STACCINI Pascal \\
\hline M. & THOMAS Pierre \\
\hline M. & TROJANI Christophe \\
\hline M. & VENISSAC Nicolas \\
\hline
\end{tabular}

Pédiatrie (54.01)

Pédopsychiatrie (49.04)

Cytologie et Histologie (42.02)

Anatomie - Chirurgie Générale (42.01)

Cancérologie - Génétique (47.02)

Pédiatrie (54.01)

Rhumatologie (50.01)

Médecine Interne (53.01)

Anesthésiologie réanimation (48.01)

Hématologie et Transfusion (47.01)

O.R.L. (55.01)

Radiologie et Imagerie Médicale (43.02)

Chirurgie Plastique (50.04)

Cardiologie (51.02)

Cancérologie ; Radiothérapie (47.02)

Thérapeutique (48-04)

Endocrinologie, Diabète et Maladies métaboliques (54.04)

Bactériologie-Virologie (45.01)

Gériatrie (48.04)

Cancérologie ; Radiothérapie (47.02)

Chirurgie Thoracique et Cardio-Vasculaire (51.03)

Anesthésiologie et Réanimation Chirurgicale (48.01)

Dermato-Vénéréologie (50-03)

Epidémiologie, Economie de la Santé et Prévention (46.01)

Maladies Infectieuses ; Maladies Tropicales (45.03)

Pédiatrie (54.01)

Bactériologie - virologie (45.01)

Endocrinologie, Diabète et Maladies Métaboliques (54.04)

Biostatistiques et Informatique Médicale (46.04)

Neurologie (49.01)

Chirurgie Orthopédique et Traumatologique (50.02)

Chirurgie Thoracique et Cardio-Vasculaire (51.03)

PROFESSEUR DES UNIVERSITES

M. SAUTRON Jean-Baptiste Médecine Générale 


\section{MAITRES DE CONFERENCES DES UNIVERSITES - PRATICIENS HOSPITALIERS}

Mme ALUNNI-PERRET Véronique

M. BENIZRI Emmanuel

M. BENOLIEL José

Mme BERNARD-POMIER Ghislaine

M. BREAUD Jean

Mme BUREL-VANDENBOS Fanny

M. DELOTTE Jérôme

M. DOGLIO Alain

Mme DONZEAU Michèle

M. FOSSE Thierry

M. FRANKEN Philippe

M. GARRAFFO Rodolphe

M. GIUDICELLI Jean

Mme HINAULLT Charlotte

Mlle LANDRAUD Luce

Mme LEGROS Laurence

M. MAGNE Jacques

Mme MAGNIE Marie-Noëlle

Mme MUSSO-LASSALLE Sandra

M. NAÏMI Mourad

M. PHILIP Patrick

Mme POMARES Christelle

Mlle PULCINI Céline

M. ROUX Christian

M. TESTA Jean

M. TOULON Pierre
Médecine Légale et Droit de la Santé (46.03)

Chirurgie Générale (53.02)

Biophysique et Médecine Nucléaire (43.01)

Immunologie (47.03)

Chirurgie Infantile (54-02)

Anatomie et Cytologie pathologiques (42.03)

Gynécologie-Obstétrique (54.03)

Bactériologie-Virologie (45.01)

Biologie du Développement et de la Reproduction (54.05)

Bactériologie-Virologie-Hygiène (45.01)

Biophysique et Médecine Nucléaire (43.01)

Pharmacologie Fondamentale (48.03)

Biochimie et Biologie Moléculaire (44.01)

Biochimie et Biologie Moléculaire ( 44.01)

Bactériologie-Virologie (45.01)

Hématologie et Transfusion (47.01)

Biophysique et Médecine Nucléaire (43.01)

Physiologie (44.02)

Anatomie et Cytologie pathologiques (42.03)

Biochimie et Biologie moléculaire (44.01)

Cytologie et Histologie (42.02)

Parasitologie et Mycologie (45.02)

Maladies Infectieuses ; Maladies Tropicales (45.03)

Rhumatologie (50.01)

Epidémiologie-Economie de la Santé et Prévention (46.01)

Hématologie et Transfusion (47.01)

\section{PROFESSEURS ASSOCIES}

M. DIOMANDE Mohenou Isidore

M. HOFLIGER Philippe

Mme POURRAT Isabelle

Mme. KLEEFIELD Sharon
Anatomie et Cytologie Pathologiques

Médecine Générale

Médecine Générale

Médecine Légale

\section{MAITRES DE CONFERENCES ASSOCIES}
M. GARDON Gilles
Médecine Générale
M. PAPA Michel
Médecine Générale

\section{PROFESSEURS CONVENTIONNES DE L'UNIVERSITE}

$\begin{array}{ll}\text { M. } & \text { BERTRAND François } \\ \text { M. } & \text { BROCKER Patrice } \\ \text { M. } & \text { CHEVALLIER Daniel } \\ \text { Mme } & \text { FOURNIER-MEHOUAS Manuella } \\ \text { M. } & \text { MAGNE Jacques } \\ \text { M. } & \text { QUARANTA Jean-François }\end{array}$

Médecine Interne

Médecine Interne Option Gériatrie

Urologie

Médecine Physique et Réadaptation

Biophysique

Santé Publique 
A ma famille, pilier fondateur et source dun bonheur infini Muriel et Clémence Papa et Maman, Morgane Papi Claude et Mamie Za

$\mathcal{A}$ la mémoire de ceux partís trop tôt, mais qui sont toujours présents 


\section{REMERCIEMENTS}

\section{Aux membres de ce jury}

A Monsieur le Professeur Philippe Paquis ; vous me faites l'honneur de présider ce jury de thèse, moment important et ô combien symbolique dans la vie d'un médecin. Vos multiples talents de chef d'équipes, d'organisateur, de neurochirurgien polyvalent font de vous un meneur que l'on suit les yeux fermés. Merci de m'avoir accueilli dans votre service et de votre enseignement au quotidien.

A Monsieur le Professeur Hugues Duffau ; vous m'avez fait l'honneur de diriger ce travail de thèse. Les six précédents mois passés à vos côtés ont été riches en enseignement qui dépasse les limites de la chirurgie, et laisseront sur moi une empreinte indélébile. Je ne vous remercierais jamais assez pour ce que vous m'avez apporté. Un grand MERCI également pour votre présence aujourd'hui.

A Monsieur le Docteur Denys Fontaine ; tu me fais l'honneur de juger ce travail de thèse. Tes qualités humaines et intellectuelles ont fortement influencé mon choix de spécialité. Notre passion commune pour cette discipline s'est rapidement transformée en une amitié solide. C'est un immense plaisir que de te côtoyer au quotidien et de suivre ton enseignement.

A Madame le Docteur Christine Lebrun-Frenay; vous me faites l'honneur de juger ce travail de thèse. Vos compétences dans les domaines abordés ici font de vous un juge incontournable. C'est un grand plaisir de recevoir votre enseignement.

A Monsieur le Professeur Michel Lonjon; vous me faites l'honneur de juger ce travail de thèse. Votre dextérité et votre charisme imposent le respect. Vos enseignements clinique et théorique sont précieux et vous n'en êtes pas avare. Merci également de m'avoir accueilli au sein de l'équipe. 


\section{A mes maîtres d'internat}

Stéphane Litrico, Antonio Iannelli, Xavier Carpentier, David Rasendrarijao, Pierre Thomas

\section{A mes aînés}

Lydiane Mondot, Guillaume Boniface, Stéphane Chanalet, Emilie Chan Seng, Luc Bauchet, Thomas Roujeau, Syril James, Domenico Ciampi, Khaled Youssef, Véronique Bourg, Caroline Giordana, Maël Launay, Marc Frenay

\section{A mes « frères, sœurs, cousins, cousines » de l'internat}

Luc Terreaux, Nathalie Chivoret, Florent Pennes, Fréderic Obadia, Julien Montoro, Nassim Bougaci, Antoine Gennari, Aurélie Leplus « Choupi », Moussa Diallo, Saad Charbek, Nathalie Vincent, Florent Desecures, Marie Manfiotto

\section{A mes amis scientifiques}

David Debruyne « Davoup », Thierry Virolle, Laurent Turchi, Mohamed Fareh, Stéphanie Begelli, Olivier Clatz

\section{A mes amis de la fac}

Michaël Collin, Vincent Meyer, Anne-Sophie Shneck, Damien Massalou, Nicolas Durand Emeline Castela, Caroline Lepeytre, Nicolas Demaneuf

A toutes les équipes soignantes des services Niçois et Montpelliérain

Avec lesquelles j'ai beaucoup de plaisir à travailler

\section{Aux anesthésistes-réanimateurs de neurochirurgie}

Richard Chemla, Gilles Rezzadori, Anne-Cécile Roux, Isabelle Petit et Emmanuelle Aguinet Aux personnes exceptionnelles qui ont permis, entre autre, à ce travail d'aboutir Guillaume Herbet et Sylvie Moritz-Gasser

\section{A mes amis}

David, Anne-So et sa maman Claude, mon filleul Simon et Hanna Moszkowicz, Fabien Gautier « La Bomba », Julien Mariani « Le GrodJ », Jean-Charles, Barbara et Emma Ragnini, Stéphanie, Kevin, Lilou et Soline Reveyrand

\section{A la famille Laffon et apparentés}

Stéphane et Elen, Barbara et Eloi, Fabrice, Laura et leurs enfants

\section{A ma famille}

Les Almairac, Giaccone, Romani, Roux, et Ricord 
Le faisceau occipito-frontal inferieur sous-tend la voie ventrale sémantique directe du langage : une approche multimodale 
SOMMAIRE

I. Introduction..................................................................p4

1. Les gliomes diffus de bas grade (GDBG)................................p4

a. Généralités

b.Histoire naturelle des GDBG

c. Prise en charge thérapeutique

$$
\begin{aligned}
\text { i. } & \text { Chirurgie } \\
\text { ii. } & \text { Radiothérapie } \\
\text { iii. } & \text { Chimiothérapie }
\end{aligned}
$$

2. Lien entre GDBG et langage..........................................p15

3. Langage et évolution des concepts....................................p16

4. Bases neurales du traitement du langage...............................p18
a. La période localisationniste
b.Du localisationnisme à l'associationnisme
c. L'émergence des réseaux parallèles et distribués

II. Objectifs de l'étude............................................................................................p24

III. Résultats (article soumis) ...................................................................................p25

IV. Principaux résultats et conclusion..............................................................p42

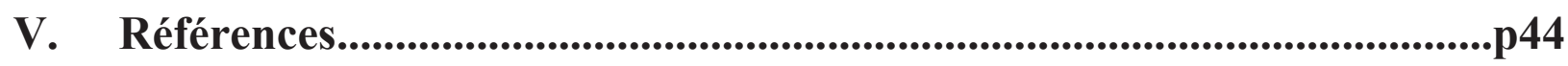

VI. Figures et tableaux..........................................................................................p54

VII. Serment d'Hippocrate.................................................................................p55 


\section{Liste des abréviations}

EmC : Extreme Capsule

FA/AF : Faisceau Arqué/Arcuate Fascicle

FLI/ILF : Faisceau Longitudinal Inférieur/Inferior Longitudinal Fascicle

FLM/MdLF : Faisceau Longitudinal Moyen/Middle Longitudinal Fascicle

FOFI/IFOF : Faisceau occipito-Frontal Inférieur/Inferior Fronto-Occipital Fascicle

FU/UF : Faisceau Unciné/Uncinate Fascicle

GDBG/DLGG : Gliome Diffus de Bas Grade/Diffuse Low-Grade Glioma

IDH: Isocitrate Déshydrogénase

IRM/MRI : Imagerie par Résonance Magnetique/Magnetic Resonance Imaging

MGMT : O6-Methylguanine-Methyltransferase

MNI : Montreal Neurological Institute

OMS/WHO : Organisation Mondiale de la Santé/World Health Organization

PCV : Procarbazine-CCNU (lomustine)-Vincristine

RT : Radiothérapie

SED/DES : Stimulation Electrique Directe/Direct Electrical Stimulation

SNC : Système Nerveux Central

TMZ : Témozolomide

VLBM : Voxel-based Lesion-Behavior Mapping 


\section{Introduction}

\section{Les gliomes diffus de bas grade (GDBG)}

\section{a. Généralités}

Les gliomes ou tumeurs gliales sont des tumeurs primitives du système nerveux central (SNC) issues du tissu de soutien ou glie. Chez l'adulte, ce sont des tumeurs rares dont l'incidence est estimée entre 6 et 8 pour 100000 habitants/an en fonction des registres [2,25]. Selon le registre national français des tumeurs primitives du SNC [125], les gliomes sont les tumeurs primitives les plus fréquentes $(42,4 \%)$ devant les méningiomes $(32,3 \%)$. Cependant, ces tumeurs constituent un groupe hétérogène, rassemblant des types et sous-types histologiques et moléculaires variés aux comportements et histoires naturelles complètement différents. La classification de l'organisation mondiale de la santé (OMS) défini 4 grades de malignité [67] (tableau 1).

\begin{tabular}{|c|c|c|c|c|c|c|}
\hline & Différenciation & $\begin{array}{l}\text { Densité } \\
\text { cellulaire }\end{array}$ & $\begin{array}{c}\text { Atypies } \\
\text { nucléaires }\end{array}$ & $\begin{array}{l}\text { Activitè } \\
\text { mitotique }\end{array}$ & Nécrose & $\begin{array}{c}\text { Prolifération } \\
\text { vasculaire }\end{array}$ \\
\hline $\begin{array}{l}\text { Grade II } \\
\text {-Fibrillaires } \\
\text {-Gémistocytiques } \\
\text {-Protoplasmiques }\end{array}$ & $\begin{array}{l}\text { Haut degré de } \\
\text { différenciation }\end{array}$ & Modérée & $\begin{array}{l}\text { Occasion- } \\
\text { nelles }\end{array}$ & $\begin{array}{l}\text { Absente } \\
\text { ou } 1 \\
\text { mitose }\end{array}$ & Absente & Absente \\
\hline $\begin{array}{l}\text { Grade III } \\
\text { Astrocytomes } \\
\text { Anaplasiques }\end{array}$ & $\begin{array}{l}\text { Anaplasie } \\
\text { focale ou } \\
\text { dispersée }\end{array}$ & $\begin{array}{c}\text { Augmentée } \\
\text { diffusément } \\
\text { ou } \\
\text { focalement }\end{array}$ & Présente & Présente & Absente & Absente \\
\hline $\begin{array}{l}\text { Grade IV } \\
\text { Glioblastomes }\end{array}$ & Faible & Elevée & Marquées & Marquée & Présente & Présente \\
\hline
\end{tabular}

Tableau 1 : critères histologiques de classification des gliomes (OMS)

Le gliome de grade I (astrocytome pilocytique) est une tumeur «bénigne» essentiellement rencontrée chez l'enfant, dont l'évolution est lente et strictement locale. L'exérèse chirurgicale complète permet la guérison dans la majorité des cas. A l'opposé, le gliome de grade IV ou glioblastome, est la tumeur primitive de SNC la plus agressive. La 
survie médiane de patients sélectionnés qui ont reçu un traitement maximaliste par chirurgie, chimio-radiothérapie concomitante et chimiothérapie d'entretien ne dépasse pas 15 mois [110]. Entre ces deux cas de figure, les comportements tumoraux sont variables ; ainsi il est préférable de séparer les «gliomes diffus de bas grade » (regroupant les grades II de l'OMS), des gliomes de haut grades (grades III et IV OMS).

\section{b. Histoire naturelle des GDBG}

Les GDBG sont des tumeurs infiltrantes du SNC qui migrent progressivement le long des faisceaux de substance blanche (fibres en « $U$ », voies d'association, commissurales, ou de projection), et ne constituent donc pas une masse tumorale bien individualisée [70]. L'évolution est chronique, systématique, et spontanée, avec une croissance linéaire pendant la phase pré-cancéreuse estimée entre 2 et $6 \mathrm{~mm} / \mathrm{an}$ (médiane à $4 \mathrm{~mm} / \mathrm{an}$ ) de diamètre tumoral moyen [86]. Sur le plan clinique, il s'agit le plus souvent de sujets jeunes (30-40 ans) dont la qualité de vie n'est pas ou peu altérée. Le mode de révélation le plus fréquent de ces tumeurs est l'épilepsie (90\%). Des bilans neuropsychologiques approfondis retrouvent des troubles cognitifs chez plus de $80 \%$ des malades, portant essentiellement sur les fonctions exécutives, la mémoire de travail, l'attention et la concentration [113-114].

Ces tumeurs évoluent inéluctablement vers la transformation anaplasique, c'est-à-dire vers un gliome malin qui va entrainer des déficits fonctionnels avec une altération rapide de la qualité de vie et le décès à court terme.

L'évolution tumorale est influencée par des facteurs intrinsèques et extrinsèques à la tumeur. Le seul facteur extrinsèque connu est la grossesse, avec une augmentation de la vitesse de croissance tumorale au cours de la gestation $[85,87]$.

Parmi les facteurs intrinsèques indépendants, on distingue :

1/ La croissance tumorale. La transformation anaplasique est d'autant plus précoce que la croissance tumorale - évaluée par la mesure répétée du volume tumoral sur des IRM successives et convertie en diamètre tumoral moyen [69] - est rapide. Dans la série du réseau Français d'étude des gliomes (REG) de 380 patients suivis pour un GDBG, en analyse uni- et multivariée, une croissance spontanée du diamètre tumoral moyen supérieure à $8 \mathrm{~mm} / \mathrm{an}$ était de façon statistiquement significative en rapport avec une diminution du délai moyen de transformation maligne (103 mois vs. 39 mois, $p<0,001)$ et de la survie globale $(249$ mois vs. 91 mois, $\mathrm{p}<0,001$ ) [89]. 
2/ Le volume tumoral. Dans cette même série, le volume tumoral était également significatif. Le délai moyen de transformation maligne et la survie globale étaient de 93 mois et de 249 mois pour un volume tumoral $<100 \mathrm{cc}$ et de 48 mois et 142 mois pour un volume tumoral $\geq$ $100 \mathrm{cc}(\mathrm{p}=0,001$ et $\mathrm{p}=0,002)$.

3/ La prise de contraste. L'apparition d'une prise de contraste au sein de la tumeur au cours du suivi ou une prise de contraste nodulaire sont corrélés à une transformation anaplasique [84].

4/ Les facteurs cytogénétiques et moléculaires. Une délétion complète du bras court du chromosome 1 ou une co-délétion de $1 \mathrm{p} / 19 \mathrm{q}$ sont associés à une évolution spontanée plus lente des GDBG. Une surexpression de p53 est au contraire associée à une évolution spontanément plus péjorative de ces tumeurs [51,95]. Les mutations pour l'isocitrate déshydrogénase 1 \& 2 (IDH) ainsi que la méthylation de l'O6-methylguaninemethyltransferase (MGMT) ne semblent pas significativement corrélées à une modification de l'évolution spontanée des GDBG [51].

5/ Les données histologiques. Le phénotype oligodendroglial pourrait avoir un meilleur pronostic que les sous-types astrocytaires ou mixtes [92]. Cependant, ces résultats ne sont pas retrouvés par tous les auteurs [61,66,89]. Dans la série des 380 patients publiée en 2013 par le REG, le sous-type histologique et le taux de prolifération n'avaient pas d'influence ni sur la vitesse de croissance des GDBG ni sur le délai moyen de transformation anaplasique.

Les débats à ce sujet sont en grande partie expliqués par l'hétérogénéité des GDBG. Les données histologiques, bien que renforcées par l'apport de la biologie moléculaire, ne représentent qu'une partie de l'ensemble des facteurs à prendre en compte dans le suivi d'un GDBG. Une autre limitation du diagnostic anatomo-pathologique vient de la nature du prélèvement. Il s'agit souvent d'une partie restreinte de la tumeur (biopsie ou exérèse partielle) qui peut ne pas intéresser un foyer anaplasique et donc sous-estimer son agressivité [83]. Une exérèse maximale de ce type de lésions (sub-totale ou totale) avec analyse anatomopathologique de l'intégralité du tissu réséqué permet d'obtenir un diagnostic histologique et un classement tumoral plus fiables [99].

Par conséquent, la classification OMS ne semble pas adaptée pour prédire à l'échelon individuel (et collectif) le comportement de ces tumeurs. Le suivi d'un patient porteur d'un GDBG se doit d'être multidimensionnel, en prenant en considération les critères statiques et dynamiques afin d'avoir une vision plus précise des facteurs pronostiques à l'échelon individuel. 
c. Prise en charge thérapeutique

Il est maintenant communément admis que les GDBG sont des tumeurs évolutives précancéreuses qui doivent être traitées dès le diagnostic établi (ou fortement suspecté sur au moins 2 IRM successives espacées de 6 semaines minimum). L'attitude de surveillance clinico-remnologique d'un GDBG - en attendant une transformation anaplasique pour commencer le traitement - est à proscrire. Une étude Norvégienne en population récente [54] a comparé les résultats de survie globale de 2 cohortes de patients en fonction de leur orientation de soin. L'attitude de l'hôpital A était de surveiller «activement » les patients après confirmation histologique (biopsie), alors que celle de l'hôpital B était de réaliser une exérèse tumorale première. Le recul médian était de 7 ans. La médiane de survie des patients de 1'hôpital A était de 5,9 ans (IC 95\%, 4,5-7,3), alors qu'elle n'était pas atteinte par les patients de l'hôpital B ( $\mathrm{p}=0,01)$ (figure A). Cette étude, méthodologiquement proche d'une étude randomisée puisque les patients étaient orientés vers l'hôpital A ou B en fonction de leur lieu d'habitation, démontre clairement le bénéfice oncologique à une prise en charge thérapeutique précoce des GDBG.

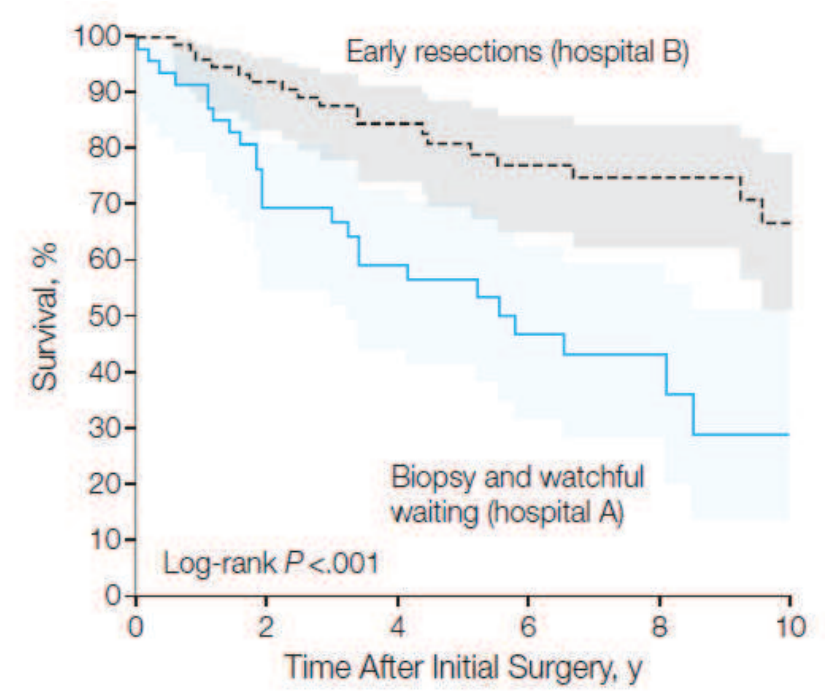

Figure A. Courbes de survie des malades en fonction de la stratégie thérapeutique adoptée.

Courbe pleine : biopsie et surveillance active (hôpital A) ; courbe pointillée : résection chirurgicale (hôpital B) (d'après Jakola et al. 2012) 
i. $\quad$ Chirurgie

La chirurgie est considérée comme le traitement le plus efficace pour retarder la transformation anaplasique et le décès [57,99].

Depuis l'article princeps de Berger et al. [6], il est établi que les résultats oncologiques dépendent de la qualité d'exérèse. Dans cette étude, une exérèse complète, intéressant tout l'hypersignal T2 à l'IRM, était corrélée à une absence de récidive tumorale pendant la durée du suivie (médiane de 41,7 mois). Pour les autres patients, l'importance du volume résiduel était corrélée à une augmentation du taux de récidive et à un raccourcissement du délai de transformation anaplasique. Ainsi, les patients avec un volume tumoral résiduel de moins de $10 \mathrm{~cm}^{3}$ (exérèse subtotale) avaient un taux de récidive de $14,8 \%$ et un délai de transformation anaplasique de 50 mois, alors que ceux qui avaient eu une exérèse partielle de leur tumeur (résidu $>10 \mathrm{~cm}^{3}$ ) avaient un taux de récidive de $46,2 \%$ et un délai moyen de transformation de 30 mois $(\mathrm{p}=0,002)$.

Ces résultats ont ensuite été confirmés par de nombreuses études [12,33,77,97,106] qui se sont également intéressées à la survie globale. En 2008, dans une série de 216 GDBG, Smith et al. ont démontré l'impact de la qualité d'exérèse tumorale sur la survie globale (figure B). Une exérèse tumorale complète était associée à une survie à 8 ans de $98 \%$ des patients $(\mathrm{HR}=$ 0.972 ; IC 95\%; p < 0,001) [106]. L'étendue de l'exérèse, lorsqu'elle était incomplète (subtotale ou partielle), était significativement corrélée à l'augmentation de la survie et au délai avant transformation anaplasique. Une exérèse d'au moins $80 \%$ de la tumeur demeurait un facteur prédictif de survie par rapport à une exérèse de moins de $80 \%$ (HR $=0.894$; IC $95 \% ; p=0,016)$. 


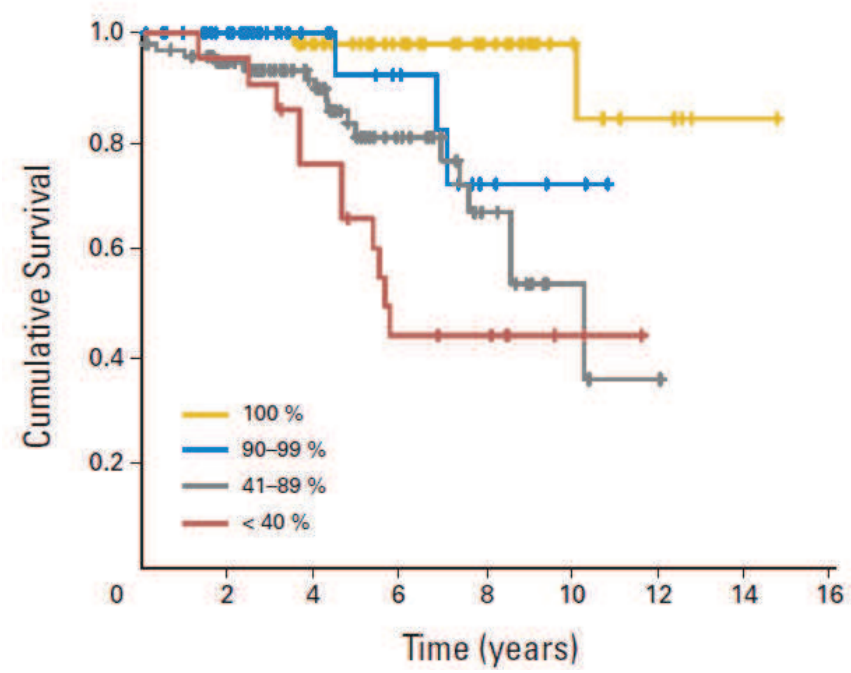

Figure B. Courbes de survie des malades en fonction de l'étendue de l'exérèse chirurgicale. L’importance de l'exérèse tumorale était associée à une amélioration de leur survie $(p<0,001)$ (d'après Smith et al. 2008)

Yordanova et al. ont introduit la notion d'exérèse supra-totale, c'est-à-dire au-delà de l'hypersignal FLAIR à l'IRM [123]. En effet, des études basées sur des prélèvements étagés péri-tumoraux ont démontré l'existence de cellules tumorales jusqu'à $20 \mathrm{~mm}$ au-delà des anomalies de signal visibles à l'IRM [88]. En comparaison à un groupe contrôle qui avait eu «seulement » une exérèse complète, l'exérèse supra-totale a permis de retarder le délai de récidive et de ne traiter qu'un patient sur les 15 par chimiothérapie adjuvante (contre 10 patients sur les 29 du groupe contrôle, $p=0,043)$. Au cours du suivi de l'étude, il n'a pas été constaté de transformation anaplasique dans le groupe "supra-total», contre 7/29 dans le groupe contrôle $(p=0,037)$. Ce type d'exérèse ne prétend pas guérir les patients d'un GDBG mais semble retarder au maximum le délai de transformation anaplasique et l'administration d'un traitement adjuvant [123].

Malgré l'étendue de la résection chirurgicale, même si elle est supra-totale, la récidive tumorale est systématique. En l'absence de transformation anaplasique, la croissance tumorale du résidu est similaire à celle de la tumeur d'origine [73]. Grâce aux mécanismes de plasticité cérébrale induits par la tumeur elle-même et par la réorganisation fonctionnelle provoquée par un geste chirurgical $[31,43]$, une ou plusieurs ré-interventions sont possibles. Une nouvelle intervention, après un délai suffisant de quelques années (en moyenne 4,1 ans dans l'étude de Martino et al.), peut permettre l'exérèse secondaire d'un résidu tumoral laissé volontairement en place lors de la première chirurgie pour des raisons fonctionnelles [74]. Certaines études 
ont démontré qu'une nouvelle exérèse d'un GDBG, lorsqu'elle est possible, est un facteur prédictif indépendant de survie globale $[1,12,74]$.

Sur le plan fonctionnel, la résection maximaliste d'un GDBG permet un contrôle de l'épilepsie dans 70 à $80 \%$ des cas [19,28,35,41,107].

Les GDBG sont des tumeurs lentement évolutives qui concernent essentiellement des sujets jeunes avec une vie normale. Ces tumeurs sont infiltrantes (sans limite nette avec le parenchyme sain) et volontiers localisées dans des zones cérébrales dites «fonctionnelles », intéressant particulièrement l'aire motrice supplémentaire et l'insula [30]. Il existe une importante variabilité anatomo-fonctionnelle interindividuelle [120], mais aussi intraindividuelle du fait des mécanismes de plasticité cérébrale induits par le développement lent et progressif de la tumeur [24,31]. Nous avons vu précédemment que l'exérèse chirurgicale est l'option thérapeutique la plus efficace actuellement, sur les plans oncologique et fonctionnel, à condition de réaliser une exérèse suffisante (supra-totale, totale ou subtotale) tout en respectant au mieux la qualité de vie des malades. L'intégration de ces paramètres onco-fonctionnels a eu pour effet de modifier la prise en charge chirurgicale des GDBG. Grâce à l'apport des techniques de stimulation cérébrale électrique directe (SED) chez des patients éveillés en cours d'intervention, il est possible de réaliser de façon fiable et reproductible une cartographie corticale et sous-corticale individuelle [39]. Cette technique a permis de considérablement améliorer les résultats onco-fonctionnels dans la chirurgie tumorale infiltrante (gliomes de haut et bas grades) [21,33] et d'augmenter les indications, notamment en zones «éloquentes » redéfinissant totalement la notion « d'opérabilité » de ces tumeurs. L'objectif est de reséquer le maximum de parenchyme cérébral envahi par la tumeur en s'arrêtant aux structures cruciales pour la fonction, à la fois aux niveaux cortical et souscortical, sans prendre de marges de sécurité [42].

Une méta-analyse récente regroupant plus de 8000 patients opérés d'un gliome (bas ou haut grade) a démontré que l'utilisation de la cartographie per-opératoire par SED permettait une réduction significative du taux de déficits post-opératoires permanents (3,4\% vs. 8,2\% sans SED) malgré l'augmentation du taux de résection en zones « éloquentes » (99,9\% vs. 95,8\% sans SED). La qualité d'exérèse a également été améliorée de façon significative, avec 75\% d'exérèse totale avec SED, contre 58\% sans SED [21] (figure C).

De plus, une série récente de l'équipe de San Francisco (UCSF) portant sur 281 malades a démontré que la cartographie per-opératoire par SED de patients dont les GDBG envahissaient des zones présumées éloquentes permettait d'augmenter significativement la survie globale (HR = 2,9, IC 95\%) grâce à une amélioration de la qualité de résection [20]. 


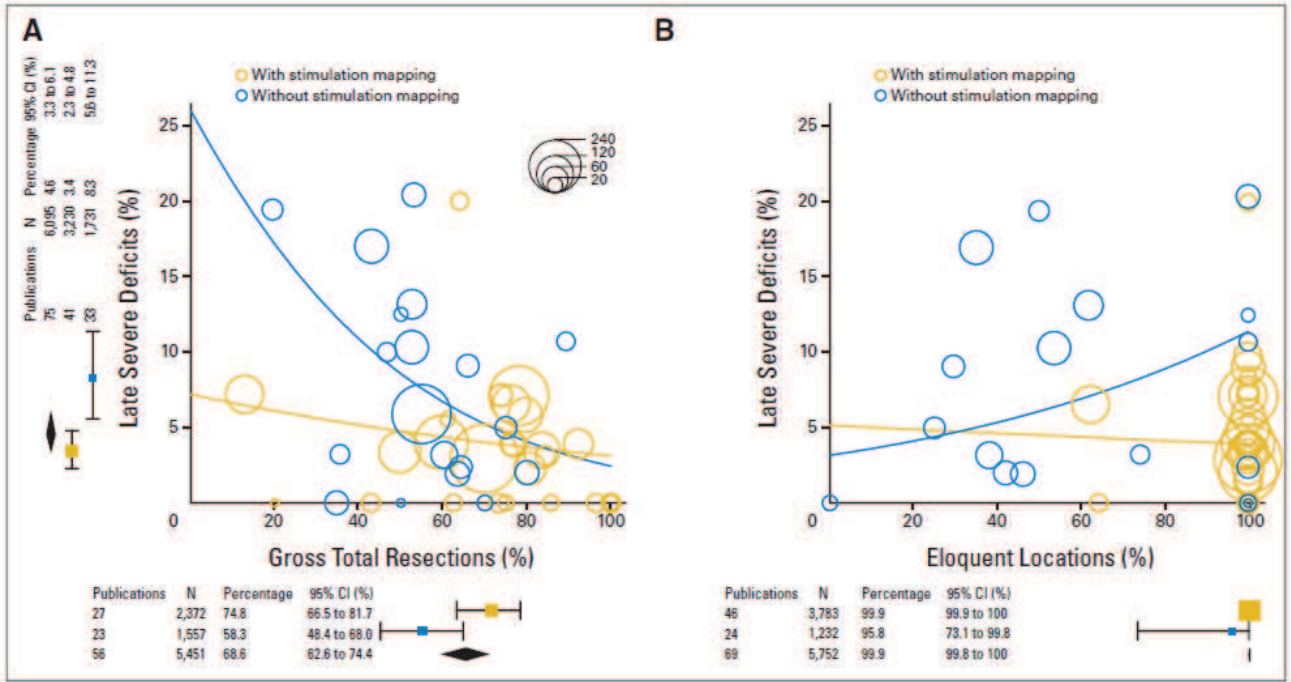

Figure C. Résultats de la chirurgie des gliomes sans (cercles bleus) et avec (cercles dorés)

SED. A. Relation entre les résultats oncologiques (\% de résection, en abscisse) et neurologiques ( $\%$ de déficits neurologique définitifs, en ordonnée) après résection tumorale.

B. Relation entre les localisations tumorales éloquentes (\%, en abscisse) et les résultats neurologiques ( $\%$ de déficits neurologique définitifs, en ordonnée) après résection tumorale.

La taille des cercles est proportionnelle à la taille des cohortes de malades des études de l'analyse. Code de couleur identique pour les courbes de régression logistique (d'après De Witt Hamer 2012)

\section{ii. $\quad$ Radiothérapie}

La radiothérapie postopératoire, que ce soit après exérèse partielle, subtotale ou totale, est longtemps restée le traitement standard d'un GDBG. Les doses utilisées sont de 50 à 54 Gy en fractions de 1,8 Gy [56,104]. Le débat persiste concernant le moment optimal d'irradiation. Une étude récente du groupe Européen de radiothérapie (EORTC) comparait l'efficacité à long terme de la radiothérapie précoce (immédiatement après la chirurgie) par rapport à une irradiation différée au moment de la progression [119]. Il n'y avait pas de différence en terme de survie globale, avec une survie médiane de 7,4 ans dans le groupe " précoce » par rapport à 7,2 ans dans le groupe contrôle $(\mathrm{p}=0,872)$. Par contre, la médiane de survie sans progression était supérieure dans le groupe précoce par rapport au groupe contrôle $(5,3$ ans vs. 3,4 ans, $p<0,0001)$. Les auteurs concluent que pour les patients avec un état neurologique satisfaisant et régulièrement suivi, la radiothérapie pourrait être différée. 
Il est désormais communément admis que la radiothérapie provoque des troubles cognitifs à long terme $[53,59,93,111]$. Une étude menée par plusieurs équipes Hollandaises sur les effets cognitifs et radiologiques à long terme de la radiothérapie à des doses dites « faibles » $(<2 \mathrm{~Gy})$ a démontré que les patients traités pour un GDBG par radiothérapie avaient de façon significative plus de déficits attentionnels, des fonctions exécutives et de la vitesse de traitement de l'information que ceux qui n'avaient pas eu de radiothérapie. Ces troubles étaient associés à des hyperdensités de la substance blanche et à une atrophie cérébrale globale [27].

Les résultats de l'étude randomisée du groupe Nord-Américain de radiothérapie (RTOG) comparant la radiothérapie seule $(\mathrm{RT})$ à la radiothérapie associée à la chimiothérapie par Procarbazine, Lomustine et Vincristine $(\mathrm{RT}+\mathrm{PCV})$ après biopsie ou exérèse d'un GDBG ont été publiés en 2012 [105]. Il n'y avait pas de différence sur la survie globale entre les 2 groupes mais une augmentation significative de la survie sans progression dans le groupe RT+PCV. Dans l'analyse post hoc il existait néanmoins un bénéfice sur la survie dans le groupe $\mathrm{RT}+\mathrm{PCV}$ réalisée à partir des survivants à 2 ans, suggérant un effet retardé de la chimiothérapie. Cependant, comme $71 \%$ des patients du groupe RT ont eu de la chimiothérapie lors de la progression tumorale, cette étude doit plutôt être considérée comme une étude comparant la chimiothérapie précoce à la chimiothérapie donnée lors de la récidive. L'ensemble de ces résultats suggèrent que la radiothérapie devrait être réservée aux patients en situation de récidive tumorale pour lesquels l'exérèse chirurgicale n'est pas envisageable, et/ou qui sont en échappement du traitement par chimiothérapie, et/ou qui présentent des signes de transformation anaplasique.

\section{iii. Chimiothérapie}

Deux chimiothérapies ont démontré leur efficacité dans les GDBG : le PCV et le témozolomide (TMZ).

Sur le plan clinique, la chimiothérapie permet une amélioration symptomatique et une réduction des crises d'épilepsie chez la plupart des patients répondeurs ou stables sur le plan tumoral [108]. Le contrôle des crises d'épilepsie est obtenu chez 50 à $80 \%$ des patients traités par chirurgie puis chimiothérapie $[63,112]$.

Sur le plan radiologique, le taux de réponse des GDBG à la chimiothérapie (PCV ou $\mathrm{TMZ}$ ), incluant les réponses mineures, est de 25 à $60 \%$ selon les critères de réponse classiques 
de McDonald (réponses complète, partielle, mineure, maladie stable, progression) $[11,49,55,95]$. Lorsque l'évaluation se fait sur le volume tumoral, le taux de réponse est compris entre $92 \%$ et $100 \%[91,95]$.

Il a été démontré que le statut moléculaire et/ou cytogénétique du gliome influence la chimiosensibilité des GDBG. La co-délétion 1p-19q est corrélée à un meilleur taux de réponse et à une réponse objective prolongée au TMZ [51,55,95]. La méthylation du promoteur de la MGMT, qui entraîne l'inactivation de cette enzyme réparatrice de l'ADN [37], et la mutation d'IDH 1 ou 2 [51] semblent être des marqueurs indépendants de chimiosensibilité.

Concernant le choix de la chimiothérapie, aucune étude comparative réalisée dans les GDBG n'est disponible. Certaines données peuvent cependant orienter le choix du clinicien. Ainsi, le TMZ est habituellement mieux toléré que le PCV dont le principal facteur limitant est la myélotoxicité. Il est également associé à une qualité de vie conservée, voire améliorée, pendant le traitement $[8,65]$. Un effet prolongé de la chimiothérapie (en première ligne de traitement) sur le volume tumoral, plusieurs mois après arrêt de celle-ci, a été démontré dans $60 \%$ des cas [91,95]. Cet effet a été évalué à 200 jours en moyenne pour le TMZ (durée moyenne de traitement de 18 mois) dans l'étude de Ricard et al., et à plus de 2 ans pour le PCV dans l'étude de Peyre et al. (figure D). Il est intéressant de noter que l'interruption prématurée du PCV (toxicité hématologique) chez 5 des 21 patients de cette étude n'a pas empêché la poursuite de la décroissance prolongée du volume tumoral (de 1 à 7 ans). Des résultats comparables ont été retrouvés dans l'étude de Lebrun et al. [63] avec un effet prolongé d'une durée moyenne de 24,4 mois après l'arrêt du PCV pour $82 \%$ des 33 patients de l'étude, avec une moyenne de 5 cycles de traitement sur les 6 initialement prévus.

Enfin, certaines équipes ont évalué la faisabilité d'une chimiothérapie néo-adjuvante lorsque l'exérèse chirurgicale première ne semble pas réalisable. C'est notamment le cas quand l'infiltration tumorale est trop diffuse (extension controlatérale, envahissement des noyaux gris centraux, de la capsule interne). L'objectif est de réduire la masse tumorale pour permettre une exérèse chirurgicale au moins sub-totale [7,109]. Même si les résultats de ces études semblent intéressants à l'échelon individuel, elles concernaient de faibles effectifs ou des cas rapportés et ne permettent pas de dégager une conduite à tenir systématique.

$\mathrm{Au}$ total, la chimiothérapie est efficace dans les GDBG en première ligne de traitement, sur les plans oncologiques et symptomatiques. L'efficacité semble corrélée au statut cyto-génétique (1p-19q) mais l'impact des marqueurs moléculaires (MGMT, IDH) n'est pas parfaitement établi. Il ne semble pas exister de différence en termes d'efficacité d'une 
chimiothérapie par rapport à l'autre (PCV ou TMZ) mais aucune étude comparative n'a été menée sur les GDBG.

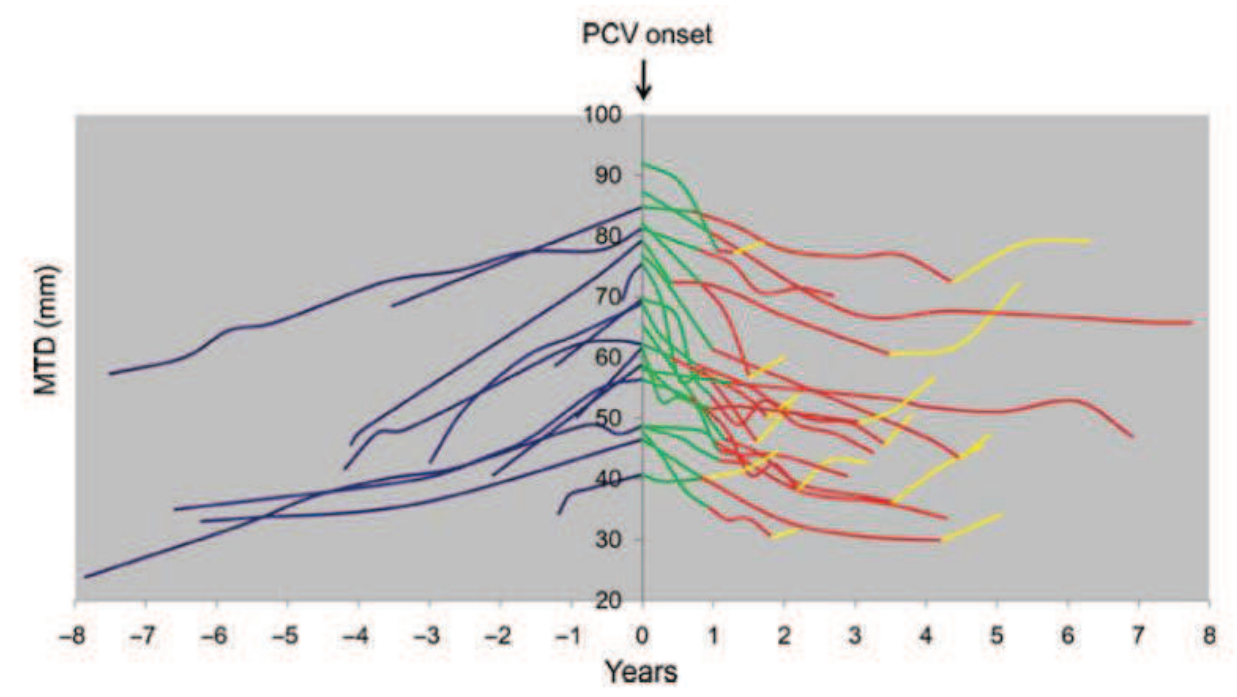

Figure D. Evolution du diamètre tumoral moyen (DTM) avant, pendant, et après chimiothérapie par PCV. Une décroissance du DTM a été observée chez tous les patients pendant la durée de la chimiothérapie. La décroissance du DTM a persisté après arrêt du PCV chez tous les patients sauf un (courbes rouges) (d'après Peyre et al. 2010) 


\section{Lien entre GDBG et langage}

Comme nous l'avons vu précédemment, les GDBG sont localisés le plus souvent dans des zones cérébrales dites «fonctionnelles », et en particulier au sein des réseaux du langage [30]. De plus, il existe une importante variabilité anatomo-fonctionnelle interindividuelle et intra-individuelle dynamique du fait des mécanismes de plasticité cérébrale induite. Nous avons également vu que le traitement chirurgical permet de modifier favorablement l'histoire naturelle de la maladie, à condition de tendre vers une exérèse tumorale satisfaisante (subtotale, totale ou supra-totale). Les techniques de SED réalisées chez des patients éveillés permettent, de façon reproductible, d'identifier les structures neurales cruciales pour le langage et de les préserver. L'exérèse tumorale se fait selon des limites fonctionnelles et non plus basée sur des examens d'imagerie pré et/ou per-opératoire dont l'intérêt pendant la chirurgie est limité par la nature infiltrante de ce type de tumeur. L'étendue de la résection tumorale est de ce fait significativement augmentée, avec un taux de déficits neurologiques significativement diminué par rapport à une exérèse effectuée sans SED [21,33].

L'expérience accumulée par les équipes qui pratiquent ce type de chirurgie a permis d'apporter des informations précieuses sur le fonctionnement du cerveau humain, et en particulier sur le langage [34]. Plus précisément, des progrès considérables ont été réalisés dans les connaissances anatomique et fonctionnelle des aires corticales et des faisceaux associatifs de substance blanche (voir chapitres suivants). C'est également l'histoire naturelle de la pathologie tumorale gliale, l'anticipation de l'évolution de ces tumeurs et des possibilités d'exérèse qui sont mieux connues grâce à l'apport de la SED. Des modèles d'évolution tumorale [70,72] ainsi que des cartes probabilistes de résidu tumoral ont été crées [52,71]. Ces connaissances de l'anatomie fonctionnelle cérébrale semblent indispensables pour tout neurochirurgien réalisant des exérèses tumorales intra parenchymateuses, en particulier au niveau sous-cortical du fait de la faible plasticité neurale au sein de la connectivité [52]. 


\section{Langage et évolution des concepts}

Le langage est une faculté proprement humaine. Il est défini par la capacité d'exprimer une pensée et de communiquer au moyen d'un système de signes: vocaux, gestuel, graphiques, tactiles, olfactifs. Le signe renvoie à une signification, alors que le signal, moyen de communication animal, à pour but de provoquer une réaction. Le langage est doté d'une sémantique (sens), et le plus souvent d'une syntaxe (structure).

Les spécificités du langage sont les capacités de double articulation (signifiant/signifié), d'alternance (communication à double sens entre les interlocuteurs), de déplacement c'est-à-dire de la capacité de parler du possible, et pas seulement du réel présent (capacité d'abstraction), de transmission culturelle spontanée, de créativité, de productivité, de métalinguistique (analyse et jugement du langage) [50]. Ainsi, si l'expérience montre que certains animaux sont capables d'exprimer leurs besoins vitaux, leurs émotions, voire même pour certains grands singes d'accéder à une expression symbolique abstraite, il n'a en revanche jamais été établi qu'un animal soit capable d'exprimer un concept ou de porter un jugement [115].

Le langage est une fonction cognitive, pas une fonction instrumentale. C'est un ensemble de représentations dont l'expression nécessite l'implication de fonctions instrumentales et motrices. Le traitement de ces différentes représentations implique d'autres fonctions supérieures telles que les fonctions mnésiques, attentionnelles et exécutives.

L'étude du langage, que ce soit pour la production (pôle expressif) ou la compréhension (pôle réceptif), implique la connaissance de ses différents niveaux de traitement. Trois niveaux de traitement sont impliqués dans les processus de compréhension et de production du mot isolé en modalité orale:

1/ le niveau phonémique, qui correspond au traitement des unités distinctives minimales de la langue, les phonèmes. Les phonèmes se combinent entre eux pour former les syllabes, puis les mots, unités significatives c'est-à-dire porteuses de sens ;

2/ le niveau lexical, qui correspond au traitement centré sur le mot. C'est le niveau où convergent les informations issues des niveaux de représentations phonémiques, sémantiques, mais aussi syntaxiques et morphologiques de traitement des mots. Les mots constituent les unités significatives minimales et sont stockés dans la mémoire à long terme sous la forme d'un lexique mental ; 
3/ le niveau sémantique, qui, d'après tous les modèles psycholinguistiques, occupe une place centrale dans le traitement du mot isolé. C'est le processus mental qui permet d'accéder au sens du mot. La mémoire sémantique contient les unités lexicales et les concepts non-verbaux, et ne fait pas uniquement référence au langage verbal, mais plus globalement aux connaissances que nous possédons sur le monde qui nous entoure [26].

Plusieurs modèles psycholinguistiques ont été proposés pour rendre compte de l'organisation cérébrale des traitements du mot. Le traitement du langage est rendu possible par l'implication d'autres fonctions cognitives. Les modèles connexionnistes de traitement parallèle et distribué permettent d'expliquer l'activation simultanée de différents niveaux de traitements, ainsi que la mise en œuvre d'un large réseau cognitif dans la production et la compréhension orale des mots. Ces modèles sont composés d'un ensemble d'unités simples de traitement interconnectées. L'activation de ces unités dépend du niveau de stimulation qu'elles reçoivent et de la force de leurs connexions. Les traitements s'effectuent en parallèle sur plusieurs unités et leurs représentations sont distribuées sur toutes les unités et leurs interconnexions [76] (figure E).

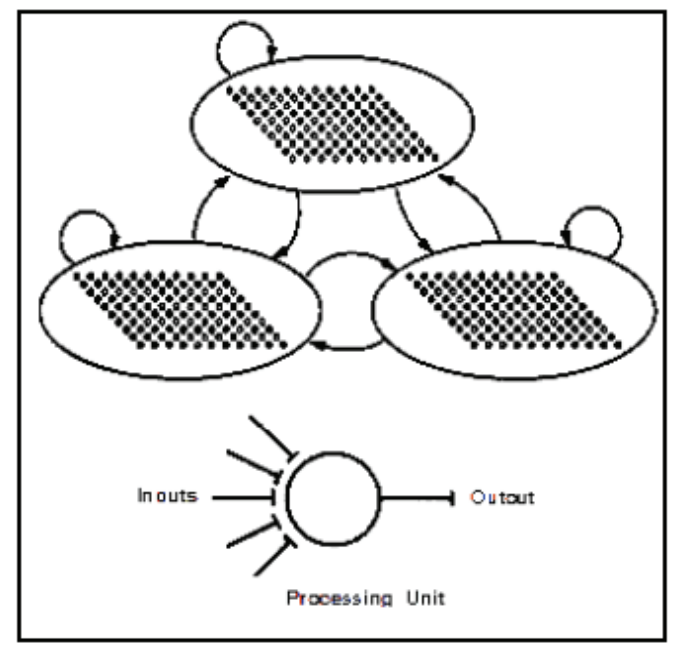

Figure E. Le modèle général de traitement parallèle et distribué (d'après Mc Clelland 1994) 


\section{Bases neurales du traitement du langage}

a. La période localisationniste

Au début du $\mathrm{XIX}^{\mathrm{ème}}$ siècle, des avancées ont eu lieu sur les bases neurales qui soustendent le langage. Les premières notions de localisation cérébrale des fonctions supérieures, le localisationnisme, sont issues de la phrénologie. L'anatomiste Allemand Franz Gall et son élève Spurzheim [124] postulaient que les bosses et affaissements du crâne, mis en évidence par palpation et craniométrie, étaient le reflet des circonvolutions cérébrales, marqueurs des facultés morales et intellectuelles. Ils mettaient en relation ces variations morphologiques avec des facultés répertoriées: calcul, langage, amitié, espoir. Ces théories pseudoscientifiques ont rapidement laissé place à l'étude du cerveau lui-même.

En 1861, Paul Broca, neurologue Français, rapportait le cas de M. Leborgne surnommé «Tan » car c'était la seule syllabe qu'il parvenait à prononcer [10]. L'autopsie de ce patient avait mis en évidence une lésion syphilitique de la partie postérieure de la troisième circonvolution frontale gauche. Broca conclut par association qu'il s'agissait du « siège de la faculté du langage articulé ». Cette zone a été baptisée « aire de Broca » (figure F).

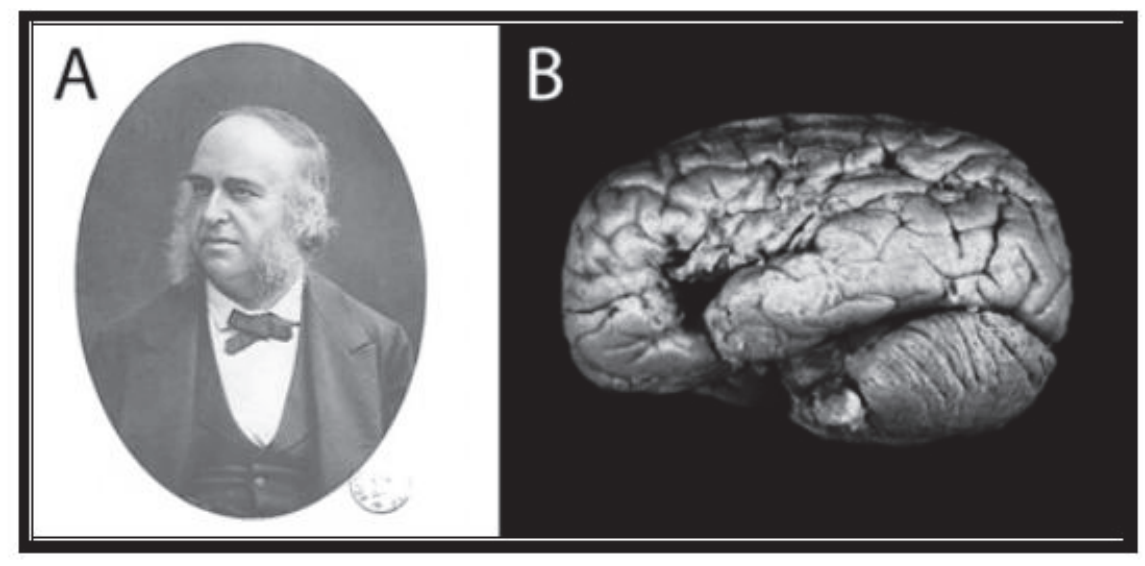

Figure F. Paul Broca et le centre de la parole. A. Portrait de Paul Broca datant de 1880. B. Hémisphère cérébral gauche de M. Leborgne (Bibliothèque Interuniversitaire Santé) 
b. Du localisationnisme à l'associationnisme

En 1874, Carl Wernicke, neurologue et psychiatre Allemand, attribuait à la partie postérieure de la première circonvolution temporale un rôle de stockage auditif des mots [121]. Cette zone cérébrale serait essentielle à la compréhension du langage. Cependant, Wernicke concevait le cerveau comme une mosaïque d'aires contenant des représentations issues d'expériences motrices (aires motrices primaires) et sensorielles (aires de l'ouïe, de la vue, de l'odorat, du goût, et aires somesthésiques). Sur la base de ce principe général, il a élaboré le premier réseau du langage (figure G); l'aire de représentation auditive était connectée directement à l'aire de représentation motrice de «Broca» via un faisceau de substance blanche en forme d'arche appelé faisceau arqué. Cette configuration permettait d'individualiser des formes d'aphasies différentes selon la localisation de l'atteinte : aphasie sensorielle, motrice ou de conduction. Ainsi était né le courant associationniste, c'est-à-dire qu'une zone cérébrale précise était reliée à une autre par un faisceau de substance blanche. Une lésion de ce faisceau induisait un syndrome de dysconnexion responsable du symptôme déficitaire.
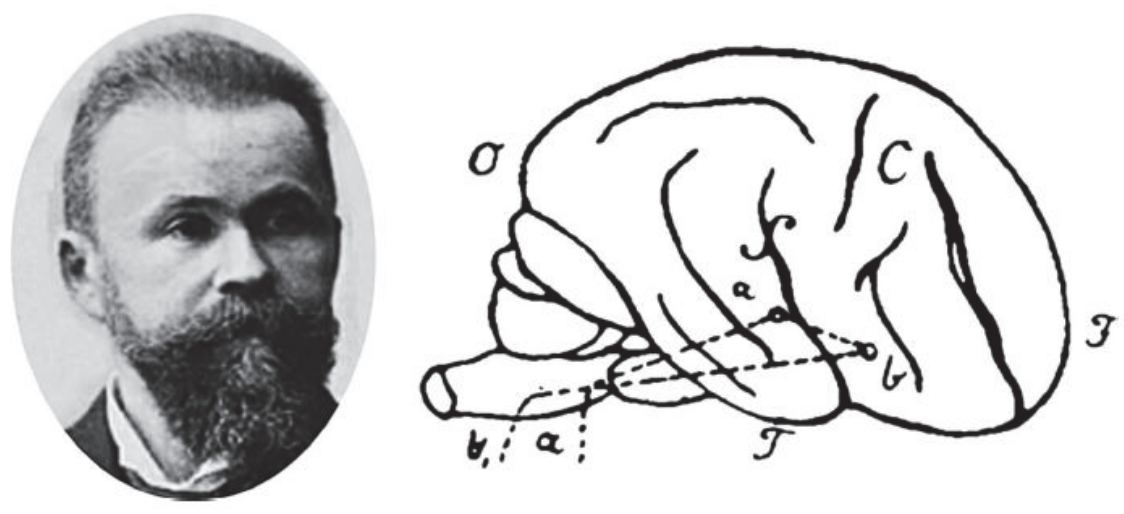

Figure G. Carl Wernicke (1848-1905) et sa représentation du réseau du langage (tiré de sa thèse de médecine, 1874) (d'après Catani et al. 2008 [17])

En 1885, Ludwig Lichtheim, neurologue Allemand, publia le schéma permettant d'illustrer le traitement cérébral du langage [64] (figure H). En s'inspirant des représentations motrices et sensorielles de Wernicke, il intercala un hypothétique "centre supérieur » qui n'était pas anatomiquement défini : le système conceptuel sémantique. Ainsi, une lésion des 
faisceaux d'association entre les différents centres corticaux (i.e. dysconnexion) permettait de décrire plusieurs types de syndromes aphasiques différents.
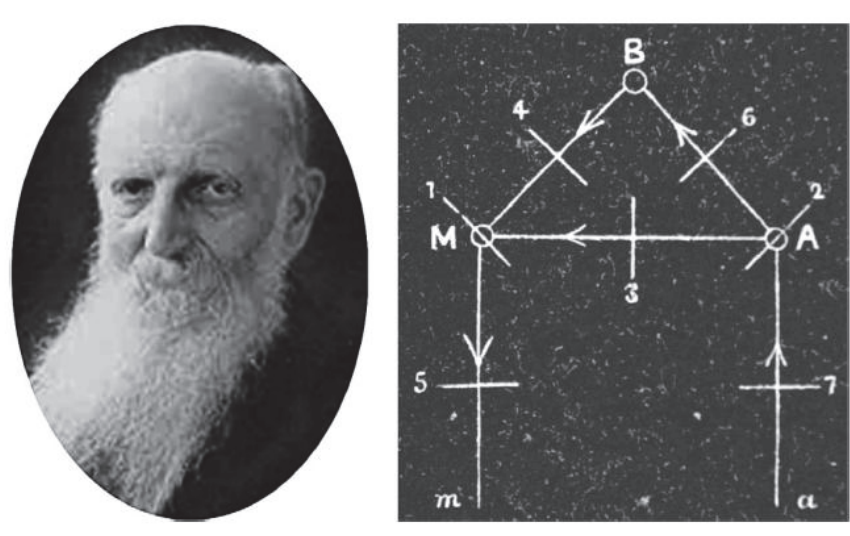

Figure H. Ludwig Lichtheim (1845-1928) et sa représentation du réseau du langage paru dans la revue Brain en 1885 (d'après Catani et al. 2008)

En 1965, Norman Geschwind, neurologue et psychiatre Américain considéré comme le père fondateur de la neurologie comportementale, publia un modèle sériel hiérarchisé inspiré de celui de Wernicke [40] (figure I). Le modèle de Geschwind-Wernicke, postulait que le traitement du langage était effectué au sein de différentes zones corticales interconnectées. La notion d'aires corticales associatives était ainsi introduite, notamment au niveau du lobule pariétal inférieur. Dans ce modèle, une lésion du cortex associatif pouvait également provoquer un syndrome de dysconnexion, au même titre qu'une lésion des voies d'association de substance blanche.
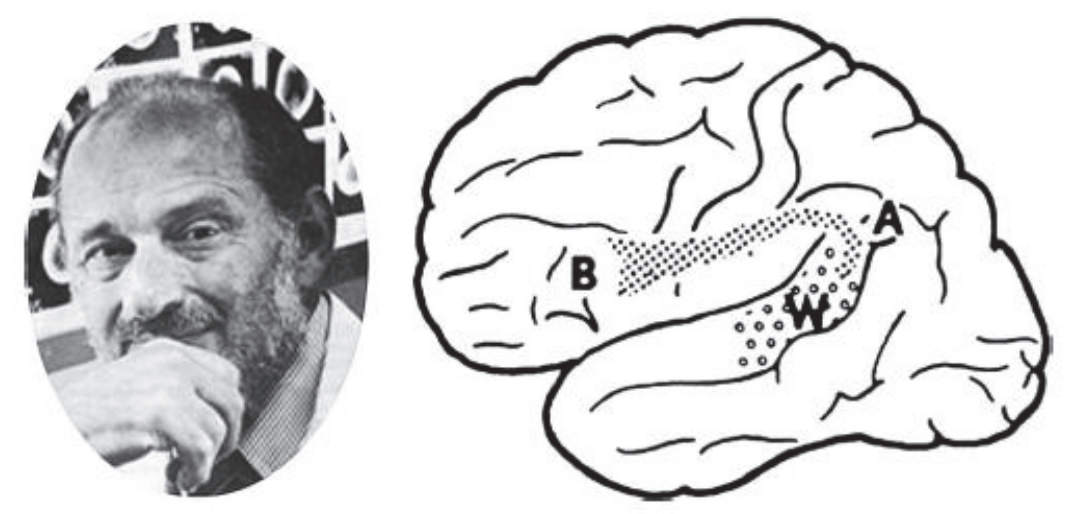

Figure I. Norman Geschwind (1926-1984) et sa représentation du réseau du langage publié dans la revue Science en 1970 (d'après Catani et al. 2008) 


\section{c. L'émergence des réseaux parallèles et distribués}

Dans les années 1980, Marsel Mesulam, neurologue et psychiatre Américain, a introduit la notion de réseaux cérébraux parallèles et distribués [78]. Il accorde alors au territoire de Geschwind (aire de «Wernicke », gyrus angulaire et supramarginal) un rôle d'intégration et d'articulation des différentes informations unimodales (liées aux sens perceptifs) permettant de les incorporer dans un réseau distribué de représentations amodales (qui n'ont aucun substrat sensoriel). Ce modèle a également introduit la notion de contrôle modulateur des réseaux attentionnels et émotionnels [79].

En 2000 et 2004, sur la base des données récentes de neuropsychologie, de psycholinguistique et de neuroimagerie, Gregory Hickok et David Poeppel ont établi un modèle d'anatomie fonctionnelle du langage [47-48]. En se basant sur les développements récents de l'organisation fonctionnelle corticale de la vision, ils ont décrits une double voie de perception du langage : la voie ventrale et la voie dorsale. La voie ventrale est impliquée dans le traitement du son en signification, et la voie dorsale dans le traitement du son en représentations articulatoires. La voie ventrale se projette ventro-latéralement, impliquant le cortex préfrontal et les régions temporales postérieures. La voie dorsale connecte le cortex pré-moteur frontal et la région parieto-temporale. Ces 2 voies sont connectées entre elles et avec les autres réseaux cérébraux formant un vaste réseau interconnecté parallèle et bidirectionnel. Malgré un certain degré de bilatéralisation, ces voies de perception du langage ont une dominance gauche.

Cependant, aussi novateur soit-il, ce modèle d'anatomie fonctionnelle du langage ne s'intéresse qu'aux structures corticales, et néglige la connectivité sous-corticale.

Depuis les années 2000, les travaux de l'équipe du Pr Hugues Duffau ont porté sur des concepts anatomiques originaux à partir de constatations per-opératoires chez des patients éveillés dans le cadre de la chirurgie tumorale cérébrale [29,33-34]. Afin de préserver les fonctions neurales tout en maximisant l'étendue de l'exérèse de gliomes diffus de bas grade (voir chapitres précédents), cette équipe réalise une cartographie corticale et sous-corticale par stimulation cérébrale électrique directe (SED). En intégrant les données issues des neurosciences, en particulier psycholinguistiques, neuropsychologiques, et de neuroimagerie fonctionnelle, avec les résultats de la SED, un schéma qui revisite les bases neurales du 
langage a été proposé [36]. Ce modèle est dit hodotopique (du grec hodos $=$ chemin, et topos $=$ lieu) en référence aux faisceaux de substance blanche qui relient des aires corticales. Il est qualifié de dynamique en référence à la plasticité cérébrale. Dans ce modèle, le langage est la résultante de traitements parallèles effectués par des groupes distribués de neurones synchronisés et connectés, en opposition à des centres individuels [34]. C'est l'interaction à grande échelle de sous-réseaux entre eux qui permet le langage, et ce jusqu'à un certain degré après lésion cérébrale ouvrant la porte aux phénomènes de plasticité cérébrale.

Dans ce modèle, les différents aspects du traitement du langage sont couverts : sémantique, phonologique et syntaxique. Il établit également un lien avec les fonctions cognitives amodales telles que la mémoire de travail et les fonctions exécutives (processus cognitifs de haut niveau permettant un comportement flexible et adapté au contexte). La figure $\mathrm{J}$ résume ce modèle.

L'entrée se fait au niveau du cortex occipital pour les informations visuelles, et au niveau de la partie postérieure du gyrus temporal supérieur pour les informations auditives. Ces influx empruntent ensuite la double voie de traitement de l'information. La voie ventrale sémantique est divisée en une voie directe, sous-tendue par le faisceau occipito-frontal inférieur (FOFI), et une voie indirecte sous-tendue par les faisceaux longitudinal inférieur (FLI) et unciné (FU). Le faisceau longitudinal moyen (FLM) aurait une fonction sémantique mais son rôle exact est encore débattu. La voie dorsale phonologique est également divisée en une voie directe via le faisceau arqué (FA) pour les aspects purement phonologiques et en une voie indirecte soustendue par la partie latérale du faisceau longitudinal supérieur (FLS). Le FLS est lui-même en lien avec la mémoire phonologique de travail et la fonction articulatoire. La sortie (production de la parole) se fait ensuite au niveau du système articulatoire qui est la voie finale commune. Ce système est représenté par le cortex prémoteur ventral, qui reçoit les afférences des 2 voies qui seront traduites en programmes moteurs articulatoires ensuite envoyés vers le cortex moteur primaire de la bouche.

Ce modèle offre de multiples avantages: i) il permet de rapprocher les schémas psycholinguistiques et neuropsychologiques à l'anatomie fonctionnelle corticale et souscorticale du cerveau ; ii) il permet d'expliquer les mécanismes de compensation sous-tendus par les phénomènes de plasticité observés après lésion cérébrale ; iii) il permet d'expliquer les dissociations cliniques du langage observées chez les cérébro-lésés ou au cours de la SED; iv) il fait le lien avec les fonctions cognitives amodales. Le lien entre ce modèle et les aspects émotionnels et comportementaux reste cependant à établir. 
Participation in

Control

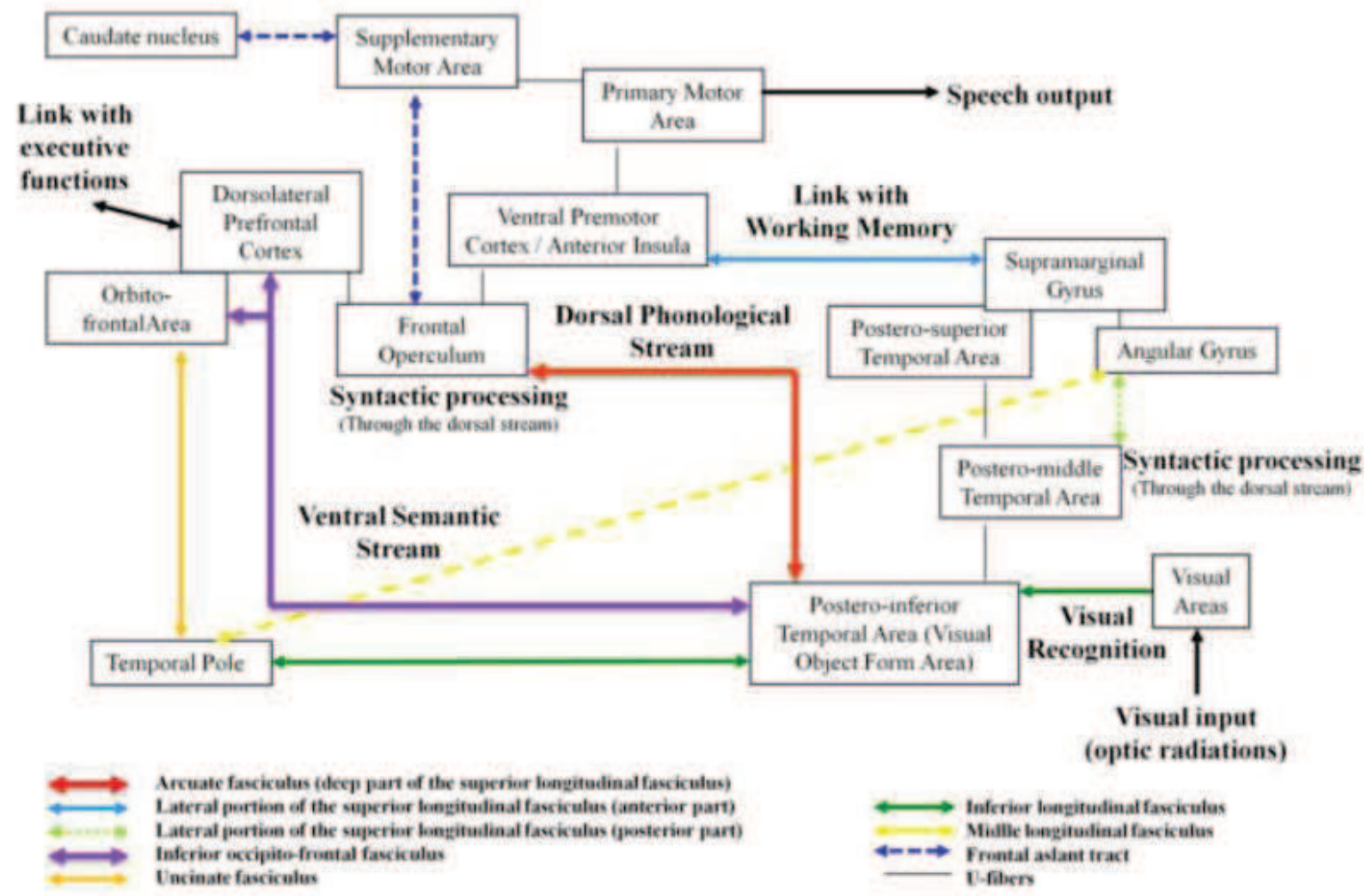

Figure J. Modèle hodotopique du langage, établi à partir de corrélations anatomofonctionnelles per-opératoires par SED (d'après Duffau et al. in press) 


\section{Objectifs de l'étude}

Les aires corticales impliquées dans le langage sont désormais bien connues. Cependant, les structures sous-corticales, et notamment les faisceaux associatifs de substance blanche, ont reçu moins d'attention. Les neuroscientifiques ont validé l'implication du faisceau arqué (FA) dans la voie dorsale phonologique mais une controverse persiste au sujet des structures neurales qui supportent la voie ventrale sémantique. Malgré les preuves accumulées en SED chez des patients éveillés de l'implication du faisceau occipito-frontal inférieur (FOFI) dans le traitement sémantique du langage [32], certains auteurs refusent d'accepter cette notion sous prétexte que le FOFI n'a pas été décrit chez le singe.

L'objectif de cette étude était de démontrer l'implication du FOFI dans le traitement sémantique du langage par une approche multi-modale.

Dans ce but, nous avons utilisé des méthodes d'association et de corrélation anatomofonctionnelle chez des malades opérés en condition éveillée d'un GDBG de l'hémisphère cérébral gauche. 


\section{Résultats}

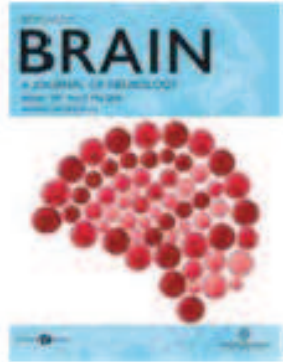

The left inferior fronto-occipital fascicle subserves language semantics: a multilevel lesion study

\begin{tabular}{|c|c|}
\hline Joumal: & Brain \\
\hline Manuscript ID: & Draft \\
\hline Manuscript Tvpe: & Oriainal Article \\
\hline Date Submitted by the Author: & $\mathrm{n} / \mathrm{a}$ \\
\hline Complete List of Authors: & $\begin{array}{l}\text { ALMAIRAC, Fabien; CHU de Nice, Hopital Pasteur, Neurosurgery } \\
\text { Heibet, Guillaume: CHU Montpellier, } \\
\text { Moritz-Gasser, Sylvie; Hôpital Gui de Chauliac, CHU de Montpellier, } \\
\text { Neurology } \\
\text { Menjot de Champfleur, Nicolas; CHU de Montpellier, } \\
\text { Duffau, Hugues; Montpellier University Medical Center, Neurosurgery ane } \\
\text { INSERM U1051 }\end{array}$ \\
\hline \multicolumn{2}{|l|}{ Key Words: } \\
\hline $\begin{array}{l}\text { Please choose up to } 5 \\
\text { kewwords from the list: }\end{array}$ & language processing, semantics, lesion studies, glioma, surgery \\
\hline
\end{tabular}

\section{SCHOY LARONE"}

Manakrah 


\section{INTRODUCTION}

Comprehension of brain language organization has radically evolved since past decades. First models were built on a localizationnist view of brain functioning $[10,121]$ with discrete cortical regions responsible for language functioning (i.e. "Broca's area" for speech production, "Wernicke's area" for auditory comprehension of language). However, as these theories could not explain all language impairments following brain damage, Lichtheim conceptually introduced the notion of interconnected cortical areas in the left hemisphere, linked with a semantic stock to produce language [64]. This was the first description of a language network, and remained for a while the main psycholinguistic model of language processing. More than 1 century later, thanks to neuroscience progress, Hickok and Poeppel have proposed a widely distributed framework of auditory language processing [47-48] and its neural basis. They integrated recent data from neuropsychological, neuroimaging, and psycholinguistic domains. Inspired by the recent description of vision neural network, they described a dual stream model of language processing. The ventral stream may be involved in the mapping of sound onto meaning (semantic processes). It connects prefrontal regions with posterior temporal areas through ventro-lateral white matter fibers. The dorsal stream connects frontal premotor and temporo-parietal regions, through dorso-lateral white matter fibers, and is involved in mapping sound onto articulatory-based representations (phonological processes). These two streams are connected each other and with other brain networks to realize an interconnected, bidirectional, and parallel distributed network. In spite of a certain degree of bilaterality, the two broad processing streams have left-sided dominance. Cortical areas involved in language are well known thanks to electrostimulation $[82,98]$ and functional imaging studies [23,120]. However, subcortical structures have received less attention. Cognitive neuroscientists have validated the involvement of the arcuate fascicle (AF) as subserving the main dorsal route $[40,101,122]$, but there is a great deal of controversy regarding the neural structures supporting the ventral semantic stream. Despite many evidences for the involvement of the inferior fronto-occipital fascicle (IFOF) in semantic processing [22,32], some authors argue that the ventral route may be supported by brain structures previously described in the macaque monkey, namely the middle longitudinal fascicle (MdLF), the uncinate fascicle (UF) and the extreme capsule (EmC) [62,90,94,101]. The IFOF has never been described in animals, only in humans. Direct electrostimulation (DES) studies in awake surgery have given compelling supports for the role of the IFOF in semantic processing (e.g. electrostimuation of the IFOF induces semantic paraphasia), 
principally in the left "dominant" hemisphere [34-35]. It connects frontal cortex (orbitofrontal, inferior and middle frontal gyrus) with posterior temporal and occipital regions. White matter path of the IFOF is well known thanks to diffuse tensor imaging (DTI) [15] and anatomical dissection studies [75]. It passes through the temporal stem above the roof of the sphenoidal horn of the ventricle, in the lower part of the extreme/external capsule.

The aim of our study was to give additional neuropsychological-based evidence for the involvement of the left IFOF as the possible semantic ventral stream. To do this, we used several methods of anatomo-functional correlations in a large sample of 31 homogeneous patients with a diffuse low-grade glioma in the left hemisphere (see Fig. 1).

A prototypical task of language assessment was used to evaluate semantic (categorical fluency) and phonological (letter fluency) processing as well.

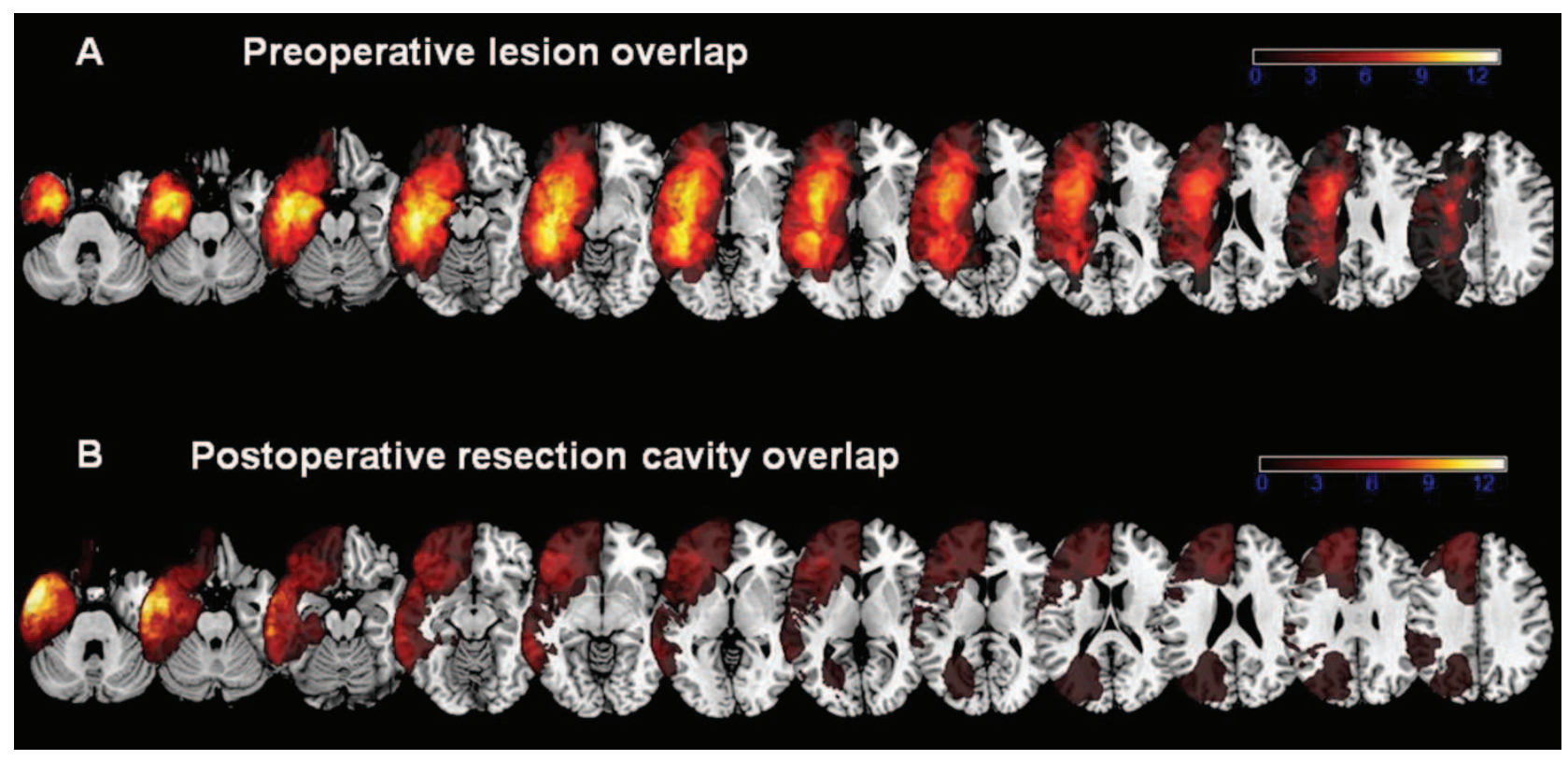

Figure 1. Lesion overlap map of the $\mathbf{3 1}$ patients in the left hemisphere. A. preoperative lesion overlap (FLAIR). B. postoperative resection cavity overlap (T1 gadolinium-enhanced). Brighter areas indicate a greater lesion overlap. 


\section{MATERIALS AND METHODS}

\section{Patients}

Thirty-one patients were recruited in this study from the medical health center of Montpellier (20 males; mean age: $35.7 \pm 9.8$ years; range: $21-54$ years). All of them were right-handed except 3 (2 left-handed and 1 ambidextrous). Educational level was $13.9 \pm 2.5$ years (range: 9-17). All Demographic data are reported in Table 1. Patients were operated on by the same senior neurosurgeon (HD) for a left supra-tentorial diffuse low-grade glioma (DLGG) according to the World Health Organization (WHO grade 2 glioma). They underwent resection surgery for the first time. Exclusion criteria were the following: (i) an age $<18$ or $>65$ years; (ii) a native language other than French; (iii) a histological examination other than WHO grade 2 glioma; (iv) a recurrent lesion previously operated. Full written consent was obtained from all patients.

\section{Behavioral testing}

Verbal fluency tests, a prototypical task of language assessment [13], were administered to patients as part of a large battery including both language and cognitive testing. This assessment was performed the day before (eval1), five days after (eval2) and three months after the surgery (eval3). Anti-epileptic treatment (if needed) was maintained constant between assessment periods. All patients benefited from rapid, intensive and specific speech rehabilitation between eval2 and eval3. In the semantic fluency task, patients are asked to generate verbal items belonging to the category of animals. In the phonological verbal fluency task, they have to generate words beginning with the letter $P$. In both category and letter fluency condition, patients are told to avoid repetitions. The time of generation is constrained to 120 seconds. Scores are given in number of correct words per $120 \mathrm{~s}$. Individual data were converted in Z-scores using control data which take into account the age and the educational level as well [13]. Scores for verbal fluency tasks are reported in Table 1. 
Table 1. Demographic data and verbal fluency $Z$-scores of all patients

\begin{tabular}{|c|c|c|c|c|c|c|c|c|c|c|c|}
\hline \multirow{2}{*}{ Patient } & \multirow{2}{*}{ sex } & \multirow{2}{*}{ Age } & \multirow{2}{*}{$\begin{array}{c}\text { Handed } \\
\text {-ness }\end{array}$} & \multirow{2}{*}{$\begin{array}{c}\text { Years of } \\
\text { education }\end{array}$} & \multirow{2}{*}{$\begin{array}{l}\text { Tumor volume } \\
\text { (voxel number } \\
0.898 \times 0.898 \times 6 \text { ) }\end{array}$} & \multicolumn{2}{|c|}{$\begin{array}{c}\text { preoperative } \\
\text { Z-scores }\end{array}$} & \multicolumn{2}{|c|}{$\begin{array}{l}\text { early postoperative } \\
\text { Z-scores }\end{array}$} & \multicolumn{2}{|c|}{$\begin{array}{l}\text { M3 postoperative Z- } \\
\text { scores }\end{array}$} \\
\hline & & & & & & sem & pho & sem & pho & sem & pho \\
\hline 1 & $\mathrm{M}$ & 31 & $\mathrm{R}$ & 9 & 66109 & $-0,58$ & 1,36 & $-4,92$ & $-2,7$ & $-2,92$ & 0,27 \\
\hline 2 & $\mathrm{M}$ & 38 & $\mathrm{R}$ & 15 & 12386 & $-0,63$ & $-2,12$ & $-2,22$ & $-3,33$ & $-0,39$ & $-0,76$ \\
\hline 3 & $\mathrm{M}$ & 51 & 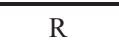 & 15 & 30368 & $-0,55$ & 0,05 & $-2,28$ & $-3,44$ & $-1,13$ & $-1,38$ \\
\hline 4 & $F$ & 42 & $\mathrm{R}$ & 15 & 29492 & $-1,13$ & 1 & -4 & $-3,92$ & $-3,54$ & $-1,7$ \\
\hline 5 & $\mathrm{M}$ & 30 & $\mathrm{R}$ & 15 & 18367 & $-1,12$ & $-0,61$ & $-3,32$ & $-3,03$ & 0,46 & $-0,61$ \\
\hline 6 & $\mathrm{M}$ & 48 & $\mathrm{R}$ & 16 & 44732 & $-0,55$ & 0,52 & $-2,28$ & $-1,86$ & $-1,7$ & 0,05 \\
\hline 7 & $F$ & 27 & $R$ & 17 & 1468 & $-0,39$ & $-0,76$ & $-1,98$ & $-1,06$ & $-0,02$ & 1,97 \\
\hline 8 & $F$ & 49 & $R$ & 15 & 4889 & 0,83 & 1,64 & $-1,36$ & 0,37 & 0,25 & $-1,06$ \\
\hline 9 & $\mathrm{M}$ & 54 & $\mathrm{~L}$ & 12 & 32759 & $-0,49$ & $-1,17$ & NA & NA & 0,25 & $-0,15$ \\
\hline 10 & $\mathrm{M}$ & 28 & $\mathrm{R}$ & 17 & 237219 & $-1,98$ & $-2,12$ & $-4,17$ & $-3,64$ & $-1,24$ & 0 \\
\hline 11 & $\mathrm{M}$ & 21 & $\mathrm{R}$ & 9 & 66251 & $-0,75$ & 0,27 & $-4,08$ & $-2,7$ & $-1,25$ & 1,52 \\
\hline 12 & $\mathrm{M}$ & 41 & $\mathrm{R}$ & 12 & 23827 & 2,16 & 2,39 & $-3,13$ & $-1,68$ & $-1,07$ & 2,56 \\
\hline 13 & $\mathrm{M}$ & 53 & $\mathrm{R}$ & 12 & 14546 & 0,69 & 1,54 & $-4,46$ & $-3,54$ & $-1,52$ & 0,86 \\
\hline 14 & $\bar{F}$ & 39 & $\bar{R}$ & 15 & 10018 & $-0,63$ & $-0,61$ & $-2,46$ & $-1,36$ & $-1,24$ & 0,15 \\
\hline 15 & $\mathrm{M}$ & 27 & $\mathrm{R}$ & 17 & 47456 & $-0,15$ & 0 & $-1,49$ & $-1,36$ & 0,22 & 0,76 \\
\hline 16 & $\mathrm{M}$ & 22 & $\mathrm{R}$ & 12 & 57456 & $-0,37$ & 0,87 & $-3,79$ & $-3,1$ & $-1,25$ & 1,19 \\
\hline 17 & $\mathrm{M}$ & 34 & A & 16 & 71121 & $-0,02$ & $-1,36$ & $-2,46$ & $-0,3$ & $-0,63$ & $-0,3$ \\
\hline 18 & $\mathrm{M}$ & 40 & $\mathrm{R}$ & 12 & 42436 & $-0,93$ & 2,05 & $-4,6$ & $-3,54$ & $-2,7$ & 0,02 \\
\hline 19 & $F$ & 30 & $\mathrm{R}$ & 15 & 171628 & $-0,88$ & $-1,36$ & $-2,46$ & $-2,42$ & $-1,73$ & $-1,21$ \\
\hline 20 & $\mathrm{~F}$ & 37 & $\mathrm{R}$ & 15 & 67450 & 1,32 & 1,82 & $-3,81$ & $-1,82$ & 0,34 & 0,91 \\
\hline 21 & $\mathrm{M}$ & 27 & $\mathrm{R}$ & 9 & 70411 & $-0,42$ & $-0,36$ & $-2,42$ & $-0,83$ & $-0,92$ & 0,89 \\
\hline 22 & $\mathrm{M}$ & 47 & $\mathrm{R}$ & 16 & 32422 & $-1,36$ & $-1,7$ & $-3,54$ & $-3,92$ & $-1,59$ & $-2,02$ \\
\hline 23 & $F$ & 23 & $\mathrm{~L}$ & 12 & 163565 & $-2,14$ & $-0,71$ & $-1,76$ & $-0,87$ & $-1,89$ & $-0,71$ \\
\hline 24 & $\mathrm{M}$ & 49 & $\mathrm{R}$ & 9 & 52343 & $-0,63$ & $-0,15$ & $-4,6$ & $-3,54$ & $-1,22$ & $-0,49$ \\
\hline 25 & $F$ & 30 & $\mathrm{R}$ & 15 & 118704 & $-1,73$ & $-1,36$ & $-2,71$ & $-2,42$ & $-1,98$ & $-1,36$ \\
\hline 26 & $\mathrm{M}$ & 41 & $\mathrm{R}$ & 15 & 13691 & 0,37 & 0,21 & $-1,47$ & $-0,9$ & $-0,78$ & $-0,59$ \\
\hline 27 & $F$ & 35 & $\mathrm{R}$ & 15 & 18768 & $-0,63$ & 0,76 & $-0,51$ & $-0,91$ & 1,2 & 0,46 \\
\hline 28 & $\mathrm{~F}$ & 32 & $\mathrm{R}$ & 12 & 20079 & 0,14 & 0,4 & $-0,25$ & $-1,67$ & 0,14 & 0,56 \\
\hline 29 & $\mathrm{M}$ & 30 & $\mathrm{R}$ & 17 & 186176 & $-1,49$ & 1,06 & $-3,68$ & $-3,03$ & $-1,96$ & $-0,61$ \\
\hline 30 & $\bar{F}$ & 23 & $\mathrm{R}$ & 14 & 36704 & $-0,63$ & $-1,21$ & $-4,17$ & $-3,64$ & 0,34 & $-1,21$ \\
\hline 31 & $\mathrm{M}$ & 27 & $\mathrm{R}$ & 16 & 60630 & $-0,76$ & 0 & NA & NA & $-0,27$ & 0,76 \\
\hline
\end{tabular}

Abbreviations: $\mathrm{A}=$ ambidextrous; $\mathrm{L}=$ left; $\mathrm{M}=$ months; $\mathrm{NA}=$ not available; pho $=$ phonologic; $\mathrm{R}=$ right; sem $=$ semantic. 


\section{Surgical procedure and intraoperative management}

All patients were operated on under local anesthesia (i.e. awake surgery) with an individual cortical and subcortical functional mapping with the use of intraoperative direct electrical stimulations (DES). DES allows the identification of neural structures involved in (language) function during tumor resection, infiltrated or not by the tumor, and consequently their preservation. This technique permits a greater extent of resection [21] thanks to tumor removal achieved until individual functional boundaries have been encountered. To perform this, language and sensory-motor skills are checked during awaken period while the neurosurgeon applies DES at the cortical and subcortical level as well (bipolar electrode, biphasic current, $60 \mathrm{~Hz}, 1.5$ to $4 \mathrm{~mA}$ intensity). DES temporarily inactivates (4 seconds) the brain region stimulated, which mimics a transient virtual lesion with a great accuracy.

Monitoring of language during surgery was performed by a speech therapist (SMG) or a neuropsychologist (GH).

\section{Imaging protocol}

Structural imaging sequences were acquired from a 1.5T (Avanto) and a 3T (Skyra) MRI Siemens scanner, the day before and three months after the surgery at the time of language assessment. We extracted from the large battery of acquisition classically performed in our center, axial FLAIR (1.5T/3T: repetition time: $13.2 \mathrm{~s} / 8 \mathrm{~s}$, echo time: $109 \mathrm{~ms} / 108 \mathrm{~ms}$, inversion time: $2.5 \mathrm{~s} / 23.7 \mathrm{~s}$, field of view: $210 \times 240 \mathrm{~mm} / 202 \times 240 \mathrm{~mm}$, slice thickness: $5 \mathrm{~mm} / 3$ $\mathrm{mm}$ and spacing: $5.5 \mathrm{~mm} / 3.6 \mathrm{~mm}$, flip angle: $150^{\circ}$ ) and axial high-resolution 3DT1-weighted gadolinium-enhanced scans (1.5T/3T: repetition time: $0.014 \mathrm{~s} / 1.7 \mathrm{~s}$, echo time: $5.2 \mathrm{~ms} / 2.54$ $\mathrm{ms}$, inversion time: $0.92 \mathrm{~s}$, field of view: 256x256 mm/250x250 mm, slice thickness: $1 \mathrm{~mm}$, flip angle: $15^{\circ} / 9^{\circ}$ ) for the following neuroanatomical analyses.

\section{Lesion segmentation and Volume of Interest (VOI) creation}

To define anatomically the lesion of each patient preoperatively, we sued the following method. Using MRIcron software (Chris Rorden, MRIcron 2012, www.mccauslandcenter.sc.edu/mricro/), lesion was first delineated on the original FLAIR MRI scanner sequence. This sequence is the most commonly used in low-grade glioma imaging because of a marked difference between healthy brain and tumor MRI signals. This first lesion segmentation was then incorporated during the normalization process to the Montreal Neurological Institute (MNI) space performed with Statistical Parametric Mapping software (SPM8, Wellcome Department of Cognitive Neurology) implemented in MATLAB 
environment (R2011a, version 7.12.0.635, www.mathworks.com). This method, known under the term of cost function masking [9], is demonstrated to limit possible biases in the registration process due to abnormal radiologic signals induced by the lesion. Then the lesion was traced again on normalized scans. A volume of interest was created for each patient. The spatial resolution was $0.898 \times 0.898 \times 6 \mathrm{~mm}$ (23 axial slices).

This method was also used to map postoperative resection cavities. However, this work was performed with 3DT1 MRI scans to improve the spatial resolution (resolution of $1 \times 1 \times 1$ $\mathrm{mm})$.

Lesion drawing was performed by $\mathrm{GH}$, a neuropsychologist with extent knowledge in neuroanatomy, who was blinded to behavioral data at the time of the work. Each VOI was compared rigorously to both original scans and detailed surgical reports of HD. A certifiedboard neuroradiologist (NMC) was asked to verify and validate the VOIs, independently. Lesion overlaps of VOI are shown in Fig. 1.

\section{Voxel-based lesion-behavior mapping (VLBM) analyses}

Voxelwise-based analyses [5] were performed using the non-parametric mapping (NPM) software [96] implemented in MRIcron package. A $t$-test for binary images and continuous behavioral variables was chosen to identify lesioned voxels associated with verbal fluency Zscores. Semantic and phonological fluencies Z-scores were treated separately. Only voxels in which at least $16 \%$ (5 individuals) of the patients had a lesion were included in the analysis. A FDR (False Discovery Rate) correction with a threshold of 5\% was chosen to correct the statistical map for multiple comparisons. The location of the most significant lesion/behavior relationship was reported with MNI coordinates.

\section{Overlapping and correlations with the IFOF and the AF}

To demonstrate a possible role of the inferior fronto-occipital fascicle (IFOF) and the arcuate fascicle (AF) in, respectively, semantic and phonological processing, we supplemented our VLBM analyses by a correlation approach. We hypothesized that decreasing performance in fluency tasks could be related to the degree of lesion infiltration of the white matter fascicles. To do this, we estimated the number of infiltrated voxels on IFOF and AF for each patient by overlapping individual lesion with the two fascicles obtained from the Catani's DTI atlas [18]. We used MRIcron to automatically compute the extent of the overlap. Results were expressed in number of infiltrated voxels for each of the two fascicles. Then, we performed Spearman 
correlation tests between verbal fluency Z-scores (semantic and phonological) and the number of infiltrated voxels for each fascicle. Statistical threshold was set at $P \leq 0.05$.

\section{RESULTS}

\section{Behavioral results}

RM ANOVAs were conducted on behavioral data. For the semantic fluency task, a main effect of the factor evaluation was revealed $[F(2,56) 46.88, P<0.000001]$. Post-hoc analyses using the Scheffé's test showed that performance dramatically decreased in the early postoperative phase (eval1 versus eval2; $P<0.001$ ) but only transiently (evall versus eval3; $P=0.082)($ Fig. 2A).

The same pattern of results was found for the phonological fluency task $[F(2,56) 50.158, P<$ 0.000001]. Performance decreased in the postoperative phase (evall versus eval2, $P<0.001$ ), but, once again, transiently (eval1 versus eval3, $P<0.92$ ) (Fig. 2B).

For both tasks, the 3 months Z-scores returned to baseline preoperative Z-scores.

A Semantic fluencytask

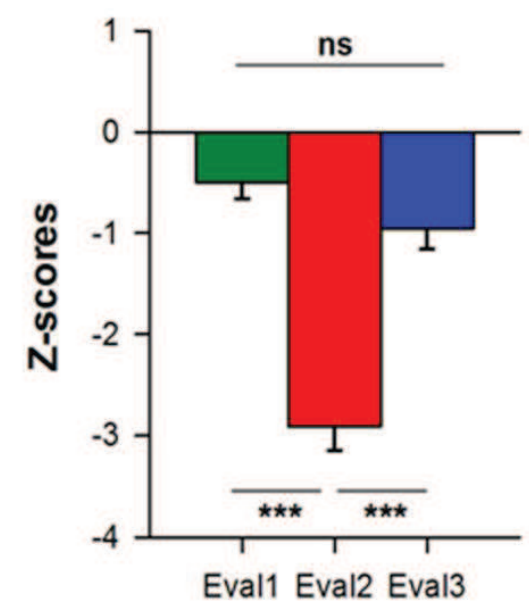

B Phonological fluencytask

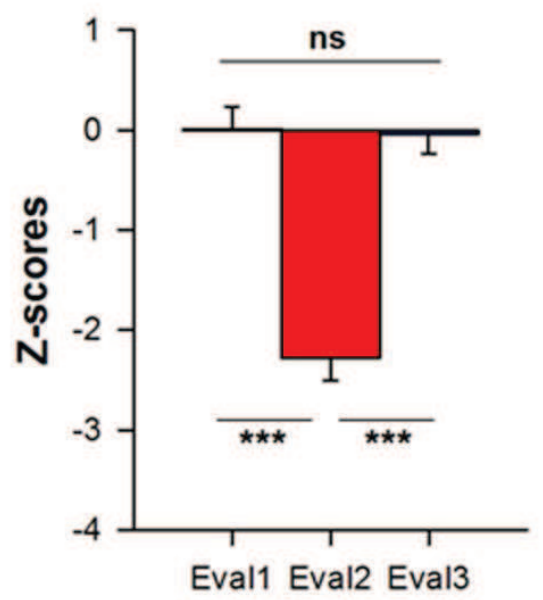

Figure 2. Histograms representing verbal fluencies $Z$-scores at the 3 periods of evaluation. A. Semantic fluency Z-scores. B. Phonological fluency Z-scores. ns $=$ non significant; $* * * P<0.001$. Eval1 $=$ preoperative evaluation; Eval2 $=\mathrm{d} 5$ postoperative evaluation; Eval3 = M3 postoperative evaluation. 


\section{Preoperative VLBM results}

The statistical map resulting from VLBM analysis on semantic fluency scores is displayed in Fig. 3A.

The largest concentration of significant voxels $\left(Z_{\text {FDR } 5 \%}=2.05\right)$ was located around the deep sylvian fissure, in the posterior orbito-frontal area (laterally and basally), in the deep white matter of the inferior frontal gyrus (iFG) and of the superior temporal gyrus (STG) along the roof of the sphenoidal horn through the limen insulae, in the deep white matter laterally to the head of caudate nucleus and to the lentiform nucleus (external capsule), and in the internal capsule (anterior and posterior limbs). A major part of these voxels corresponded to the perisylvian language area and seemed to project into the path of the inferior fronto-occipital fascicle (IFOF, see discussion section). The statistically most significant voxels ( $Z_{\text {FDR }} 1 \% \geq$ 3.07) were found in the white matter of the pars triangularis and opercularis of the $\mathrm{iFG}$, at the level of the limen insulae (MNI $\mathrm{x}=-29, \mathrm{y}=32, \mathrm{z}=4 ; Z=3.44$, cluster size $=3284$ voxels $)$ and in the deep white matter of the posterior part of the STG at the level of the entry into the sagittal stratum $(\mathrm{MNI} x=-36, \mathrm{y}=-32, \mathrm{z}=10 ; Z=3.4$, cluster size $=5414$ voxels $)$.

Regarding the phonological fluency task, no voxel reached significativity after FDR correction for multiple comparisons (Fig. 3B). 


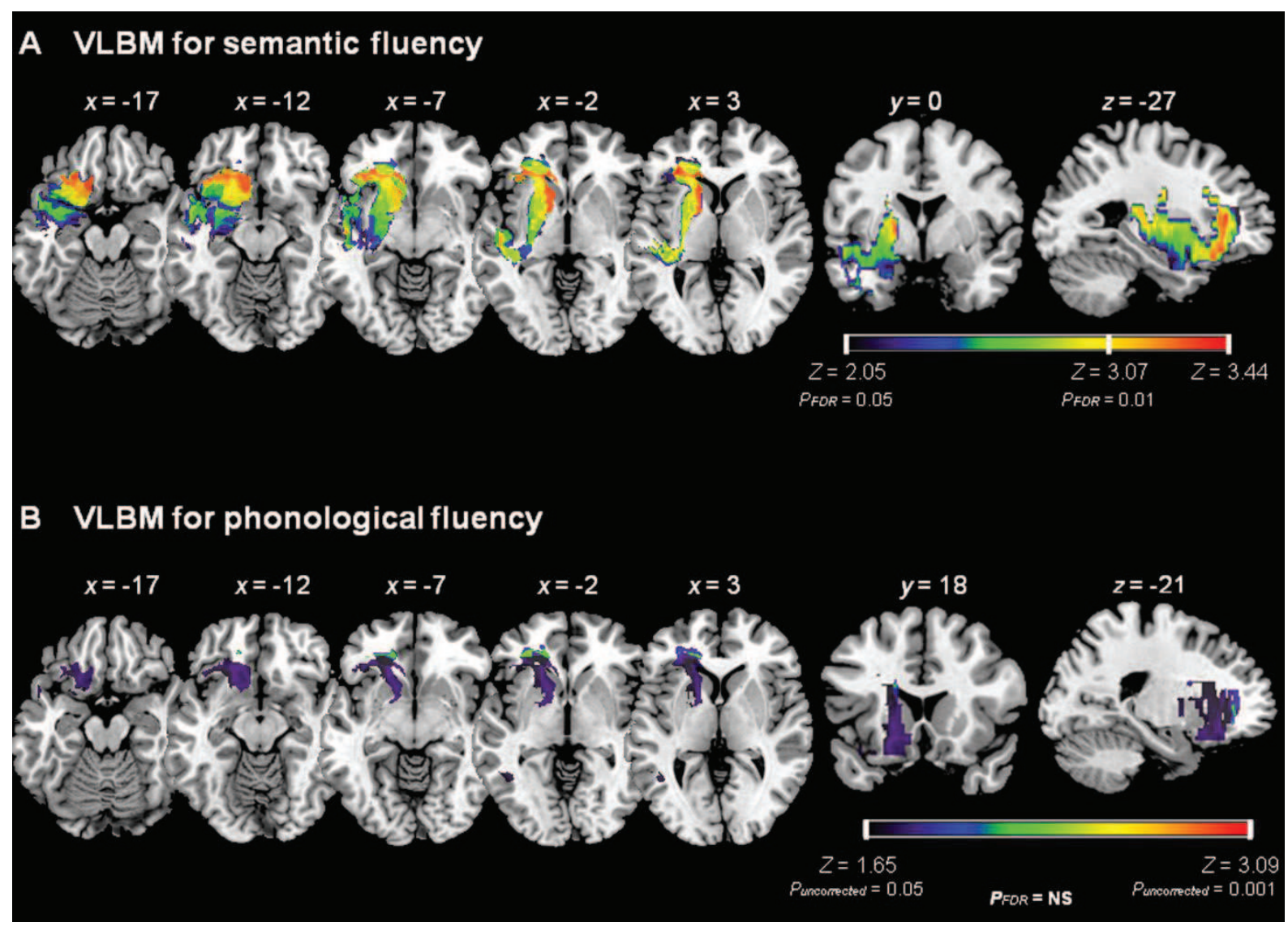

Figure 3. Preoperative statistical voxel-based lesion-behavior mapping (VLBM) analyses for verbal fluencies. Montreal Neurological Institute coordinates of each section are given, $x$ for axial slices, $y$ for coronal slices, and $z$ for sagittal slices. A. VLBM for semantic fluency task. Map shows only significant voxels with FDR-controlled threshold $(P<0.05$ for $Z=$ 2.05). B. VLBM for phonological fluency task. No voxels were significant after FDR correction $\left(P_{F D R}=\mathrm{NS}\right)$ 


\section{Overlapping and correlations with the IFOF and the AF}

As mentioned above, a large part of significant voxels found in the semantic fluency VLBM analysis seemed to overlap with the spatial location of the IFOF. To better visualize this finding, we superimposed the semantic fluency statistical map and the left IFOF obtained from the Catani's DTI atlas. As shown in Fig. 4A, the significant voxels of the statistical map overlaid with the IFOF at the level of its anterior half. Of 20515 voxels constituting the IFOF mask, 7731 (37.7\%) overlapped with the semantic fluency statistical map.

To better define this relation, we completed VLBM analyses with a correlation approach. From these analyses, it was found that semantic fluency Z-scores correlated with the volume of lesion infiltrated in IFOF $\left(r_{31}=-0.4, P=0.029\right.$, Fig. 4B).

To explore the involvement of the dorsal stream, particularly the AF, in semantic function of language, an overlap of the statistical map with left AF from the same DTI atlas was performed (Fig. 4C). Except for a little portion of the temporo-parietal part of the AF, there was no overlapping with significant voxels of the statistical map. Of 29483 voxels constituting the AF mask, 445 (1.5\%) overlapped with the semantic fluency statistical map.

The same correlation analyses were conducted. A marginal effect was found between semantic fluency Z-scores and AF infiltrated voxels $\left(r_{31}=-0.34, P=0.059\right.$, Fig. 4D).

Concerning phonological fluency task, no significant relationship was revealed for the two fascicles (IFOF: $r_{31}=-0.2, P=0.28$; AF: $r_{31}=-0.19, P=0.3$ ).

Taken together, the patterns of results presented here provide strong evidence for an essential involvement of the IFOF in semantic processing. 


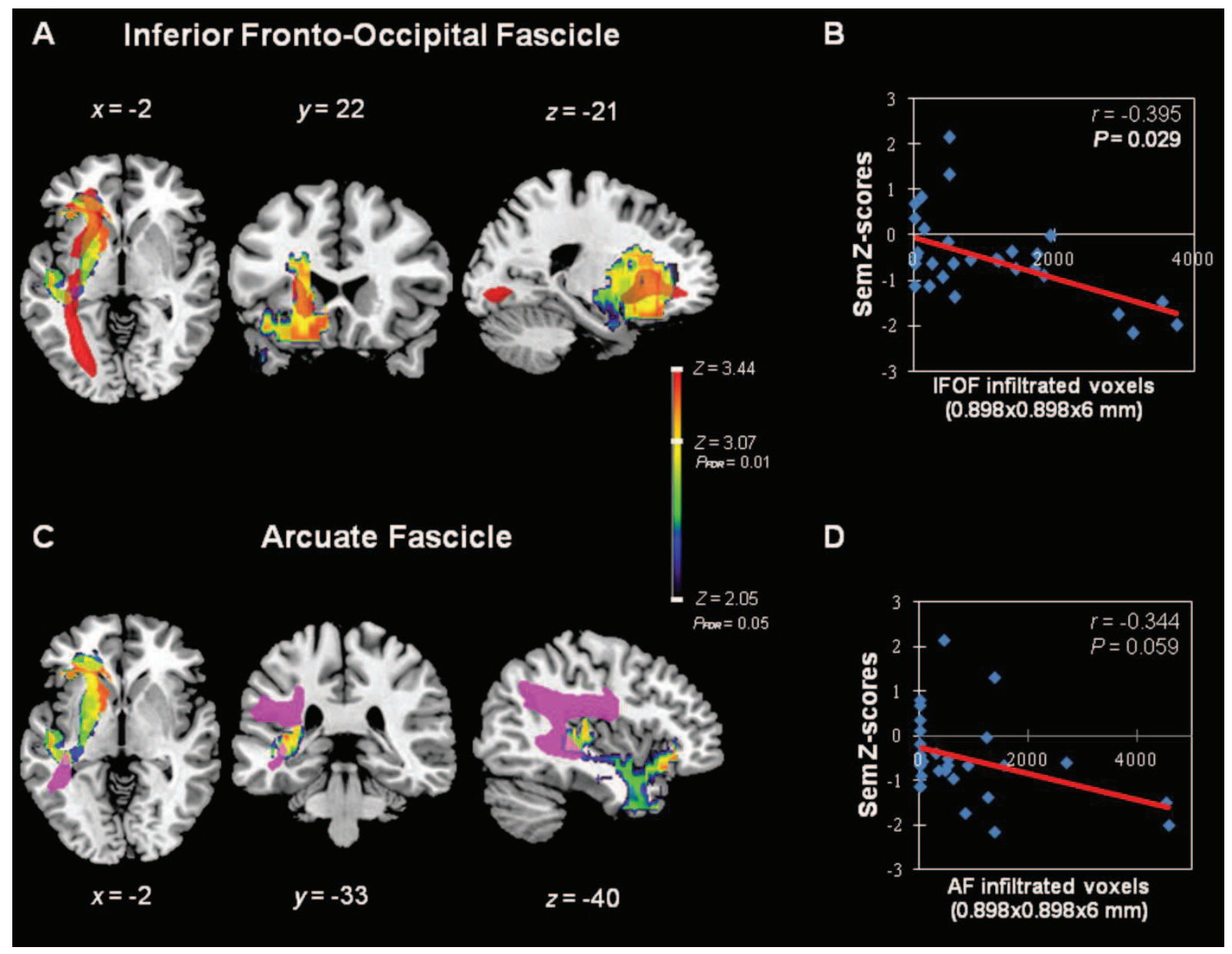

Figure 4. Overlap of ventral and dorsal stream fascicles with the preoperative VLBM map for semantic fluency and the Spearman correlation scatterplots for semantic Zscores and the two fascicles infiltrated voxels from the Catani DTI atlas. MNI coordinates of each section are given ( $x$ for axial slices, $y$ for coronal slices, and $z$ for sagittal slices). A. Overlap of IFOF (red) and preoperative VLBM map for semantic fluency. B. Spearman correlation scatterplots for semantic Z-scores and IFOF $(P=0.029)$. C. Overlap of AF (pink) and preoperative VLBM map for semantic fluency. D. Spearman correlation scatterplots for semantic Z-scores and AF ( $P=\mathrm{NS})$. 


\section{Postoperative VLBM results}

The statistical maps resulting from VLBM analyses on both early postoperative semantic and phonological fluency scores are displayed in Fig. 5A and B. No voxel reached the level of significativity after FDR correction.

The results were reproduced when considering three-month resective cavity maps.

(Fig. 5C and D).

These results indicate that there is no relation/association between the location of resection cavities and verbal fluency scores, either immediately after surgery or at the 3-months postoperative period.

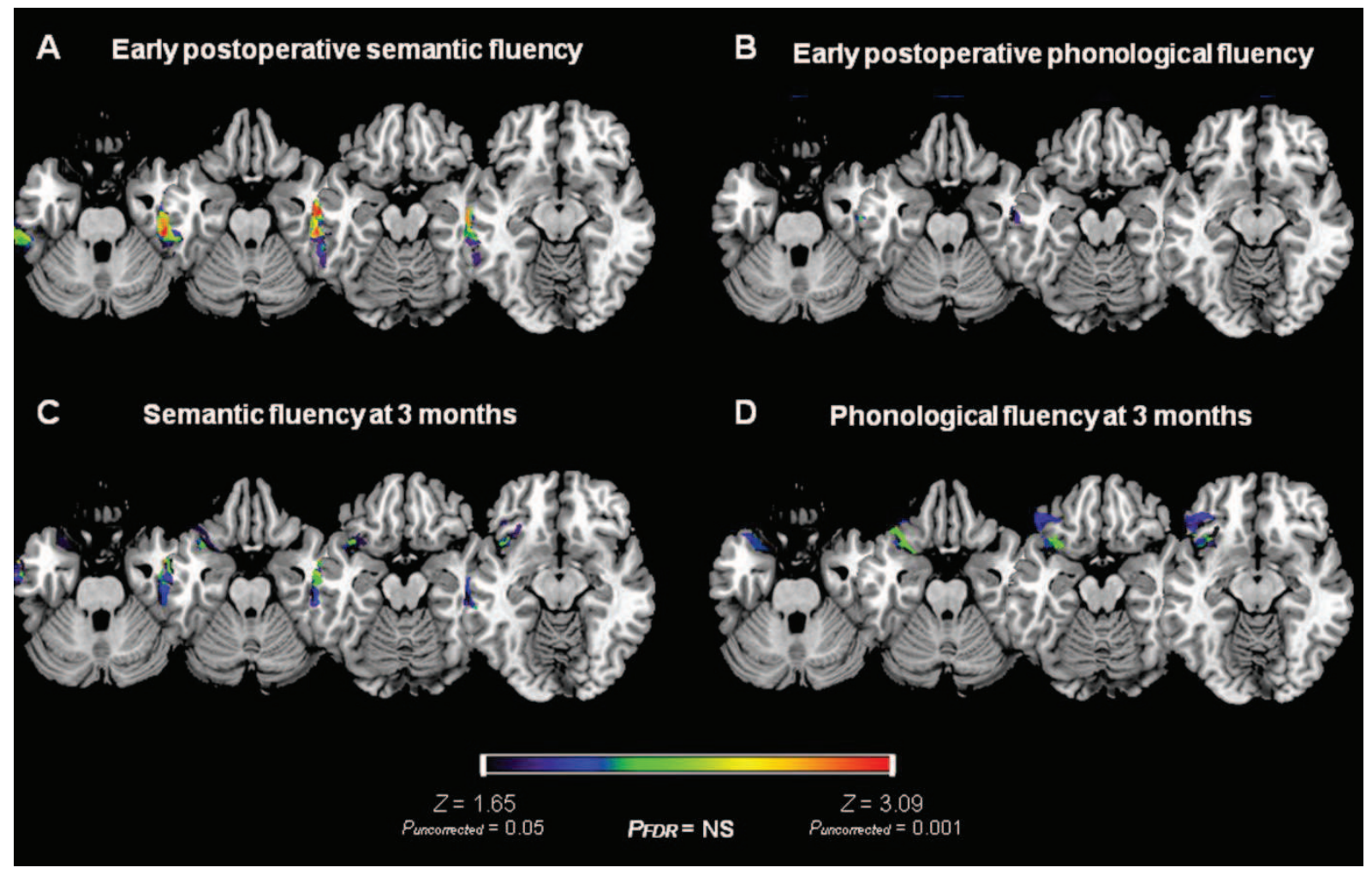

Figure 5. Postoperative cavity voxel-based lesion-behavior mapping (VLBM) analyses for verbal fluencies, at early postoperative period and 3 months after surgery. No voxels were significant for both fluency and both period tests after FDR correction $\left(P_{F D R}=\mathrm{NS}\right)$. MNI coordinates for axial slices are $x=-27, x=-22, x=-17, x=-12$. A. VLBM for early postoperative semantic fluency task. B. VLBM for early postoperative phonological fluency task. C. VLBM for 3 months postoperative semantic fluency task. D. VLBM for 3 months postoperative phonological fluency task. 


\section{DISCUSSION}

In this study, we used several techniques of anatomo-functional correlation analyses to ascertain the essential involvement of the left IFOF in the ventral semantic stream of language. The results obtained and discussed in the following confirm this hypothesis. Preoperatively, an aggregate of significant voxels was associated with the scores obtained from the semantic fluency task, especially at the level of the ventro-lateral white matter. These voxels were found to overlap to a great extent with the spatial location of the IFOF. In agreement with this, a correlation was revealed between the infiltration volumes in this fascicle and the behavioral data.

\section{Anatomical and functional correspondences with our findings}

Several studies from different (anatomical, radiological, electrophysiological) disciplines have demonstrated the existence of a human intra-hemispheric association tract which may be involved in semantic processing: the inferior fronto-occipital fascicle (IFOF) $[15,32,34,75]$.

The IFOF is a ventral associative bundle that connects the frontal lobe with the occipital and parietal. It crosses through in the depth of the temporal lobe and insula. Recent anatomical studies using post-mortem white-matter dissection according to Klingler's method [58] and DTI works have studied the main course of the IFOF. It runs at the level of the limen insulae at the posterior two-thirds of the temporal stem, it reaches the roof of the temporal horn within the anterior and middle temporal lobe at the ventral part of the external capsule, then it joins the sagittal stratum in the posterior portion of temporal stem $[38,75,117]$. In our study, the trajectory of the main subcortical portion of IFOF seems to spatially correspond to the bulk of significant voxels found at the level of the white matter for the semantic fluency task, as attested by the great overlap with the IFOF mask from Catani's DTI atlas (37.7\%).

Concerning the exact frontal terminal branches of IFOF, it remains unclear regarding to their strong intersection with other long tract terminal branches, in particular AF and superior longitudinal fascicle (SLF) [75]. Recent DTI tractography studies have described frontal connections of the IFOF with the dorso-lateral prefrontal and orbito-frontal cortex $[18,38]$. In a multiple approaches study including DTI, post-mortem dissection and intraoperative direct electrostimulation (DES), Sarubbo et al. describe two layers of the IFOF [100]. The superficial layer has frontal terminations in the $\mathrm{iFG}$ (pars triangularis and opercularis), and the deep layer has frontal terminations in the lateral and basal orbito-frontal 
cortex, medial frontal gyrus and dorsolateral prefrontal cortex. Both have cortical terminations in the so-called "Wernicke's area", namely the posterior part of the superior temporal gyrus (pSTG), and in the fusiform area, the superior parietal lobule and occipital cortex. In our study, we found significant voxels in the pars triangularis of the $\mathrm{iFG}$, in the lateral and basal orbito-frontal cortices, and in the pSTG. It may correspond to the cortical terminations of IFOF previously described.

However, regarding to the preferential temporo-fronto-insular distribution of lesions in our series, we were not able to explore the possible involvement of the posterior part of IFOF,

including basi-temporal, parietal and occipital cortical terminations. As a consequence, only the anterior half of IFOF and its frontal and pSTG cortical terminations are represented in our analyses.

DES studies have allowed an improved knowledge of language connectivity organization $[29,32]$. Combining the language disturbances elicited by stimulations with the anatomical data provided by pre- and post-operative MRI, allows to perform reliable anatomo-functional correlations especially with regard to the language pathways [46]. In such a condition, it is shown that DES of IFOF induces reproducible semantic paraphasias, whatever the part of the bundle stimulated [32,35], whereas DES of AF induces reproducible phonological paraphasias [29]. These observations reported in our study give additional support for the involvement of IFOF in semantic processing. The degree by which the IFOF is infiltrated is correlated with semantic fluency task scores.

\section{Behavioral testing and marginal effect on AF for semantic fluency}

In the current study, verbal fluency task was used as a classical assessment of language processing. These tests are commonly proposed to assess executive functioning, as they require efficient organization of verbal retrieval and recall as well as self-monitoring aspects of cognition, self-initiation, and inhibition of inappropriate responses. It constitutes a useful validated assessment to differentiate both level of language processing: phonological and semantic $[13,45]$. Most of functional and behavioral studies showed that category-fluency task requires a strategic search through semantic memory requiring left temporal lobe involvement, whereas letter-fluency task involves preferentially the phonological memory through left frontal regions involvement $[4,44,81]$.

However, some authors have demonstrated a possible lack of discrimination between the two processes concerning patients with frontal lesions $[3,45]$. It could be due to a failure 
in executive function resulting in an impairment of strategic search through memory. Furthermore, semantic processes can be involved in both fluency tasks [103]. Fluency responses may be constrained by the semantic features of words generated, not only during category fluency, but also during letter-fluency task. Nevertheless, even if an interaction between both levels of processing is necessary in both conditions of word generation (phonological and semantic), it remains that phonological fluency involves mainly the phonological level of language processing while semantic fluency involves mainly the semantic level of language processing.

Concerning the dorsal stream of language processing, it is increasingly recognized that the AF supports the phonological processes and that the superior longitudinal fascicle (SLF) supports the articulatory processes [16,29,34,68,101-102]. Surprisingly, we found a marginal effect in correlation analyses between semantic fluency Z-scores and total number of AF infiltrated voxels $(r=-0.34, P=0.059)$, whereas a lower correlation effect was found regarding the phonological fluency Z-scores $(r=-0.19, P=0.3)$. This apparent contradictory result could be due to: (i) a discrete impairment of executive functions for frontal tumor location explaining lower results (behavioral and correlation) for semantic Z-scores than for phonological ones; (ii) the fact that DLGGs in frontal region might infiltrate both IFOF and AF where fibers intersect at the level of iFG [100] (i.e. the close relationship between the ventral and dorsal stream might induce a confounding effect for patients of our study) wrongly suggesting the involvement of AF in semantic processing; (iii) the inhomogeneous tumor spatial distribution: lesions are more represented in the temporo-insular regions (i.e. ventral route) than in posterior temporal and parietal region (i.e. dorsal route), that can lead preferentially to a functional disruption of the ventral compared to dorsal connectivity.

\section{Subcortical connectivity and hodotopical brain organization}

With respect to the behavioral data, analyses have shown that, although a significant decrease of performance was observed for both fluency tasks, this was only transient. This finding fits well with those others studies which show that severe but transient worsening can be noted in the immediate postoperative phase, at the neurologic and cognitive level as well [114]. Possible explanations of this transient worsening are (i) transient edema and not actual brain injury [14]; (ii) brain plasticity and dynamic anatomo-functional organization of the brain [31]. In this latter case, several patterns of functional reshaping has been demonstrated $[24,60]$. 
The brain hodotopical organization (i.e. large-scale distributed networks at the whole brain level) may explain the absence of significant relationship between postoperative resective cavity maps and behavioral data, even in the early postoperative period. As critical cortico-subcortical structures in the language networks have been preserved during surgery thanks to individual intraoperative functional mapping, VLBM analyses could not associated resected voxels with behavioral impairment. Furthermore, the Z-scores kinetics (i.e. the transient immediate postoperative worsening but a 3 months postoperative recovery) strengthen the fact that neural structures mandatory for language function, particularly the subcortical connectivity, have been identified (and then preserved) during surgery. The originality of this study, compared to stroke studies, is that we can demonstrate with a great accuracy the involvement of subcortical connectivity by a multilevel approach, using a combination of DES findings with a voxelwise-based analysis.

In sum, our findings give news insights on the current debate about the connectional anatomy of the ventral semantic stream. Specifically, our overall results highlight the left inferior fronto-occipital fascicle as a pivotal structure. This does not call in question the possible role of other ventral white matter fibers in semantic processing, as the UF, the MdLF, or the inferior longitudinal fascicle (ILF), as argued by other authors [90,94,101,118]. In fact, in the most recent integrative models of language neural organization, authors describe a double ventral semantic stream, composed of a direct pathway (IFOF) and an indirect pathway (UF and ILF, with a possible involvement of MdLF) [36]. The present study, which was not designed to stress the issue of the indirect pathway, underlines the essential involvement of the left IFOF in the direct pathway. 


\section{Principaux résultats et conclusion}

Dans cette étude, à partir de différentes méthodes d'association et de corrélation anatomofonctionnelle chez des malades opérés en condition éveillée d'un GDBG de l'hémisphère cérébral gauche, nous avons montré que le FOFI est probablement impliqué dans la voie ventrale sémantique du langage.

En pré-opératoire, les voxels infiltrés par la tumeur significativement associés aux scores de fluence sémantique étaient localisés au niveau de la substance blanche ventro-latérale $(\mathrm{p}<0,05)$. Ces voxels étaient superposables avec 37,7\% de ceux du FOFI de l'atlas radiologique de Catani. De plus, une corrélation négative entre les scores de fluence sémantique et le nombre de voxels infiltrés du FOFI était mise en évidence $(r=-0,4, p=0,029)$. Il n'y avait pas de corrélation ni d'association significative avec les scores phonologiques ou avec ceux impliquant le FA, ce qui démontre la spécificité du FOFI pour les aspects sémantiques du langage.

En post-opératoire, il n'y avait pas de relation statistique entre les cavités de résection et les scores de fluence verbale. De plus, les scores de fluence (sémantique et phonologique) à 3 mois étaient identiques à ceux obtenus en pré-opératoire, avec un déficit transitoire constaté lors de l'évaluation post-opératoire immédiate. Ces résultats renforcent la notion d'organisation cérébrale hodotopique du langage, c'est-à-dire que des zones corticales fonctionnelles sont interconnectées par des faisceaux d'association indispensables à la fonction. Le FOFI et le FA ont été identifiés par SED (déficit transitoire des scores de fluence), et préservés (récupération à 3 mois).

Les résultats de cette étude apportent des arguments supplémentaires impliquant le FOFI au centre des processus sémantiques du langage. Toutefois, comme le défendent de nombreux auteurs, le rôle dans les fonctions sémantiques du langage d'autres structures neurales telles que le faisceau longitudinal moyen, le faisceau longitudinal inférieur, le faisceau unciné ou la capsule extrême, n'est pas remis en question ici. Ces notions sont compatibles avec les modèles les plus récents d'organisation du langage. La voie ventrale sémantique comporte une voie directe, indispensable à la fonction et constituée par le FOFI, et une voie indirecte constituée par les autres faisceaux précédemment cités [36].

Il a récemment été mis en évidence que le FOFI ne serait pas seulement impliqué dans le traitement sémantique modal (verbal) du langage, mais aussi dans les processus sémantiques 
amodaux (non-verbaux) et leur conscience [80]. En d'autres termes, le FOFI permettrait de rejoindre la conscience noétique décrite par Tulving, c'est-à-dire la conscience de la connaissance et de la compréhension de l'environnement et de soi, ainsi que la conscience de cette conscience [116]. Ces résultats ouvrent la voie à une approche plus globale du langage, incluant les aspects cognitifs de haut niveau tels que les processus sémantiques amodaux ou supra-modaux. Des tests cliniques courants tels que le Pyramid Palm-Trees Test (PPTT), test d'association sémantique non-verbal, sont désormais utilisés en chirurgie éveillée afin d'identifier - et ainsi de préserver - avec plus de précision les fonctions multiples d'une structure anatomique. Des liens avec les aspects émotionnels et comportementaux restent à établir afin d'articuler l'ensemble des réseaux cérébraux et de les intégrer dans un schéma de fonctionnement global incluant tous les aspects cognitifs propres à l'Homme. 


\section{Références}

1. Ahmadi R, Dictus C, Hartmann C, et al. Long-term outcome and survival of surgically treated supratentorial low-grade glioma in adult patients. Acta Neurochir (Wien) 2009; 151: 1359-65.

2. Baldi I, Gruber A, Alioum A, et al. Descriptive epidemiology of CNS tumors in France: results from the Gironde Registry for the period 2000-2007. Neuro Oncol 2011; 13: $1370-8$.

3. Baldo JV, Shimamura AP. Letter and category fluency in patients with frontal lobe lesions. Neuropsychology 1998; 12: 259-67.

4. Baldo JV, Schwartz S, Wilkins D, Dronkers NF. Role of frontal versus temporal cortex in verbal fluency as revealed by voxel-based lesion symptom mapping. J Int Neuropsychol Soc 2006; 12: 896-900.

5. Bates E, Wilson SM, Saygin AP, et al. Voxel-based lesion-symptom mapping. Nat Neurosci 2003; 6: 448-50.

6. Berger MS, Deliganis AV, Dobbins J, Keles GE. The effect of extent of resection on recurrence in patients with low grade cerebral hemisphere gliomas. Cancer 1994; 74: 1784-91.

7. Blonski M, Pallud J, Goze C, et al. Neoadjuvant chemotherapy may optimize the extent of resection of World Health Organization grade II gliomas: a case series of 17 patients. J Neurooncol 2013.

8. Brada M, Viviers L, Abson C, et al. Phase II study of primary temozolomide chemotherapy in patients with WHO grade II gliomas. Ann Oncol 2003; 14: 1715-21.

9. Brett M, Leff AP, Rorden C, Ashburner J. Spatial normalization of brain images with focal lesions using cost function masking. Neuroimage 2001; 14: 486-500.

10. Broca P. Remarques sur le siège de la faculté du langage articulé, suivies d'une observation d'aphémie (perte de la parole). Bulletin de la Société Anatomique 1861: $330-57$.

11. Buckner JC, Gesme D, Jr., O'Fallon JR, et al. Phase II trial of procarbazine, lomustine, and vincristine as initial therapy for patients with low-grade oligodendroglioma or oligoastrocytoma: efficacy and associations with chromosomal abnormalities. J Clin Oncol 2003; 21: 251-5. 
12. Capelle L, Fontaine D, Mandonnet E, et al. Spontaneous and therapeutic prognostic factors in adult hemispheric World Health Organization Grade II gliomas: a series of 1097 cases. J Neurosurg 2013.

13. Cardebat D, Doyon B, Puel M, et al. [Formal and semantic lexical evocation in normal subjects. Performance and dynamics of production as a function of sex, age and educational level]. Acta Neurol Belg 1990; 90: 207-17.

14. Carrabba G, Fava E, Giussani C, et al. Cortical and subcortical motor mapping in rolandic and perirolandic glioma surgery: impact on postoperative morbidity and extent of resection. J Neurosurg Sci 2007; 51: 45-51.

15. Catani M, Howard RJ, Pajevic S, Jones DK. Virtual in vivo interactive dissection of white matter fasciculi in the human brain. Neuroimage 2002; 17: 77-94.

16. Catani M, Jones DK, ffytche DH. Perisylvian language networks of the human brain. Ann Neurol 2005; 57: 8-16.

17. Catani M, Mesulam M. The arcuate fasciculus and the disconnection theme in language and aphasia: history and current state. Cortex 2008; 44: 953-61.

18. Catani M, Thiebaut de Schotten M. A diffusion tensor imaging tractography atlas for virtual in vivo dissections. Cortex 2008; 44: 1105-32.

19. Chang EF, Potts MB, Keles GE, et al. Seizure characteristics and control following resection in 332 patients with low-grade gliomas. J Neurosurg 2008; 108: 227-35.

20. Chang EF, Clark A, Smith JS, et al. Functional mapping-guided resection of low-grade gliomas in eloquent areas of the brain: improvement of long-term survival. Clinical article. J Neurosurg 2011; 114: 566-73.

21. De Witt Hamer PC, Robles SG, Zwinderman AH, et al. Impact of intraoperative stimulation brain mapping on glioma surgery outcome: a meta-analysis. J Clin Oncol 2012; 30: 2559-65.

22. de Zubicaray GI, Rose SE, McMahon KL. The structure and connectivity of semantic memory in the healthy older adult brain. Neuroimage 2011; 54: 1488-94.

23. Demonet JF, Thierry G, Cardebat D. Renewal of the neurophysiology of language: functional neuroimaging. Physiol Rev 2005; 85: 49-95.

24. Desmurget M, Bonnetblanc F, Duffau H. Contrasting acute and slow-growing lesions: a new door to brain plasticity. Brain 2007; 130: 898-914.

25. Dolecek TA, Propp JM, Stroup NE, Kruchko C. CBTRUS statistical report: primary brain and central nervous system tumors diagnosed in the United States in 2005-2009. Neuro Oncol 2012; 14 Suppl 5: v1-49. 
26. Donaldson W, Tulving E. Organization of memory. 1972 Academic Press, New York.

27. Douw L, Klein M, Fagel SS, et al. Cognitive and radiological effects of radiotherapy in patients with low-grade glioma: long-term follow-up. Lancet Neurol 2009; 8: 810-8.

28. Duffau H, Capelle L, Lopes M, et al. Medically intractable epilepsy from insular lowgrade gliomas: improvement after an extended lesionectomy. Acta Neurochir (Wien) 2002; 144: 563-72; discussion 72-3.

29. Duffau H, Capelle L, Sichez N, et al. Intraoperative mapping of the subcortical language pathways using direct stimulations. An anatomo-functional study. Brain 2002; 125: 199-214.

30. Duffau H, Capelle L. Preferential brain locations of low-grade gliomas. Cancer 2004; 100: 2622-6.

31. Duffau H. Lessons from brain mapping in surgery for low-grade glioma: insights into associations between tumour and brain plasticity. Lancet Neurol 2005; 4: 476-86.

32. Duffau H, Gatignol P, Mandonnet E, et al. New insights into the anatomo-functional connectivity of the semantic system: a study using cortico-subcortical electrostimulations. Brain 2005; 128: 797-810.

33. Duffau H, Lopes M, Arthuis F, et al. Contribution of intraoperative electrical stimulations in surgery of low grade gliomas: a comparative study between two series without (1985-96) and with (1996-2003) functional mapping in the same institution. J Neurol Neurosurg Psychiatry 2005; 76: 845-51.

34. Duffau H. The anatomo-functional connectivity of language revisited. New insights provided by electrostimulation and tractography. Neuropsychologia 2008; 46: 92734.

35. Duffau H, Peggy Gatignol ST, Mandonnet E, et al. Intraoperative subcortical stimulation mapping of language pathways in a consecutive series of 115 patients with Grade II glioma in the left dominant hemisphere. J Neurosurg 2008; 109: 461-71.

36. Duffau H, Moritz-Gasser S, Mandonnet E. A re-examination of neural basis of language processing: proposal of a dynamic hodotopical model from data provided by brain stimulation mapping during picture naming. Brain and Language in press.

37. Everhard S, Kaloshi G, Criniere E, et al. MGMT methylation: a marker of response to temozolomide in low-grade gliomas. Ann Neurol 2006; 60: 740-3.

38. Fernandez-Miranda JC, Rhoton AL, Jr., Alvarez-Linera J, et al. Three-dimensional microsurgical and tractographic anatomy of the white matter of the human brain. Neurosurgery 2008; 62: 989-1026; discussion 26-8. 
39. Fontaine D, Duffau H, Litrico S. [New surgical techniques for brain tumors]. Rev Neurol (Paris) 2006; 162: 801-11.

40. Geschwind N. The organization of language and the brain. Science 1970; 170: 940-4.

41. Ghareeb F, Duffau H. Intractable epilepsy in paralimbic Word Health Organization Grade II gliomas: should the hippocampus be resected when not invaded by the tumor? J Neurosurg 2012; 116: 1226-34.

42. Gil-Robles S, Duffau H. Surgical management of World Health Organization Grade II gliomas in eloquent areas: the necessity of preserving a margin around functional structures. Neurosurg Focus 2010; 28: E8.

43. Gil Robles S, Gelisse P, Vergani F, et al. Discrepancies between preoperative stereoencephalography language stimulation mapping and intraoperative awake mapping during resection of focal cortical dysplasia in eloquent areas. Stereotact Funct Neurosurg 2008; 86: 382-90.

44. Gourovitch ML, Kirkby BS, Goldberg TE, et al. A comparison of rCBF patterns during letter and semantic fluency. Neuropsychology 2000; 14: 353-60.

45. Henry JD, Crawford JR. A meta-analytic review of verbal fluency performance following focal cortical lesions. Neuropsychology 2004; 18: 284-95.

46. Henry RG, Berman JI, Nagarajan SS, et al. Subcortical pathways serving cortical language sites: initial experience with diffusion tensor imaging fiber tracking combined with intraoperative language mapping. Neuroimage 2004; 21: 616-22.

47. Hickok G, Poeppel D. Towards a functional neuroanatomy of speech perception. Trends Cogn Sci 2000; 4: 131-38.

48. Hickok G, Poeppel D. Dorsal and ventral streams: a framework for understanding aspects of the functional anatomy of language. Cognition 2004; 92: 67-99.

49. Hoang-Xuan K, Capelle L, Kujas M, et al. Temozolomide as initial treatment for adults with low-grade oligodendrogliomas or oligoastrocytomas and correlation with chromosome 1p deletions. J Clin Oncol 2004; 22: 3133-8.

50. Hockett CF. The origin of speech. Sci Am 1960; 203: 89-96.

51. Houillier C, Wang X, Kaloshi G, et al. IDH1 or IDH2 mutations predict longer survival and response to temozolomide in low-grade gliomas. Neurology 2010; 75: 1560-6.

52. Ius T, Angelini E, Thiebaut de Schotten M, et al. Evidence for potentials and limitations of brain plasticity using an atlas of functional resectability of WHO grade II gliomas: towards a "minimal common brain". Neuroimage 2011; 56: 992-1000. 
53. Iuvone L, Mariotti P, Colosimo C, et al. Long-term cognitive outcome, brain computed tomography scan, and magnetic resonance imaging in children cured for acute lymphoblastic leukemia. Cancer 2002; 95: 2562-70.

54. Jakola AS, Myrmel KS, Kloster R, et al. Comparison of a strategy favoring early surgical resection vs a strategy favoring watchful waiting in low-grade gliomas. JAMA 2012; 308: $1881-8$.

55. Kaloshi G, Benouaich-Amiel A, Diakite F, et al. Temozolomide for low-grade gliomas: predictive impact of 1p/19q loss on response and outcome. Neurology 2007; 68: 1831-6.

56. Karim AB, Maat B, Hatlevoll R, et al. A randomized trial on dose-response in radiation therapy of low-grade cerebral glioma: European Organization for Research and Treatment of Cancer (EORTC) Study 22844. Int J Radiat Oncol Biol Phys 1996; 36: 549-56.

57. Keles GE, Lamborn KR, Berger MS. Low-grade hemispheric gliomas in adults: a critical review of extent of resection as a factor influencing outcome. J Neurosurg 2001; 95: 735-45.

58. Klinger J. Erleichterung der makroskopischen Praeparation des Gehirns durch den Gefrierprozess. Schweizer Archiv für Neurologie und Psychiatrie 1935; 36: 247-56.

59. Koot RW, Stalpers LJ, Aronica E, Andries Bosch D. Cerebral necrosis after 25Gy radiotherapy in childhood followed 28 years later by 54Gy radiotherapy. Clin Neurol Neurosurg 2007; 109: 607-12.

60. Krainik A, Duffau H, Capelle L, et al. Role of the healthy hemisphere in recovery after resection of the supplementary motor area. Neurology 2004; 62: 1323-32.

61. Kreth FW, Faist M, Rossner R, et al. Supratentorial World Health Organization Grade 2 astrocytomas and oligoastrocytomas. A new pattern of prognostic factors. Cancer 1997; 79: 370-9.

62. Kummerer D, Hartwigsen G, Kellmeyer P, et al. Damage to ventral and dorsal language pathways in acute aphasia. Brain 2013; 136: 619-29.

63. Lebrun C, Fontaine D, Bourg V, et al. Treatment of newly diagnosed symptomatic pure low-grade oligodendrogliomas with PCV chemotherapy. Eur J Neurol 2007; 14: 3918.

64. Lichtheim L. On aphasia. Brain 1885: 433-85.

65. Liu R, Solheim K, Polley MY, et al. Quality of life in low-grade glioma patients receiving temozolomide. Neuro Oncol 2009; 11: 59-68. 
66. Lote K, Egeland T, Hager B, et al. Survival, prognostic factors, and therapeutic efficacy in low-grade glioma: a retrospective study in 379 patients. J Clin Oncol 1997; 15: 312940.

67. Louis DN, Ohgaki H, Wiestler OD, et al. The 2007 WHO classification of tumours of the central nervous system. Acta Neuropathol 2007; 114: 97-109.

68. Maldonado IL, Moritz-Gasser S, de Champfleur NM, et al. Surgery for gliomas involving the left inferior parietal lobule: new insights into the functional anatomy provided by stimulation mapping in awake patients. J Neurosurg 2011; 115: 770-9.

69. Mandonnet E, Delattre JY, Tanguy ML, et al. Continuous growth of mean tumor diameter in a subset of grade II gliomas. Ann Neurol 2003; 53: 524-8.

70. Mandonnet E, Capelle L, Duffau H. Extension of paralimbic low grade gliomas: toward an anatomical classification based on white matter invasion patterns. J Neurooncol 2006; 78: 179-85.

71. Mandonnet E, Jbabdi S, Taillandier L, et al. Preoperative estimation of residual volume for WHO grade II glioma resected with intraoperative functional mapping. Neuro Oncol 2007; 9: 63-9.

72. Mandonnet E, Pallud J, Clatz O, et al. Computational modeling of the WHO grade II glioma dynamics: principles and applications to management paradigm. Neurosurg $\operatorname{Rev} 2008 ; 31: 263-9$.

73. Mandonnet E, Pallud J, Fontaine D, et al. Inter- and intrapatients comparison of WHO grade II glioma kinetics before and after surgical resection. Neurosurg Rev 2010; 33: 91-6.

74. Martino J, Taillandier L, Moritz-Gasser S, et al. Re-operation is a safe and effective therapeutic strategy in recurrent WHO grade II gliomas within eloquent areas. Acta Neurochir (Wien) 2009; 151: 427-36; discussion 36.

75. Martino J, Brogna C, Robles SG, et al. Anatomic dissection of the inferior fronto-occipital fasciculus revisited in the lights of brain stimulation data. Cortex 2010; 46: 691-9.

76. McClelland JL. The organization of memory. A parallel distributed processing perspective. Rev Neurol (Paris) 1994; 150: 570-9.

77. McGirt MJ, Chaichana KL, Attenello FJ, et al. Extent of surgical resection is independently associated with survival in patients with hemispheric infiltrating lowgrade gliomas. Neurosurgery 2008; 63: 700-7; author reply 07-8.

78. Mesulam M. Neurocognitive networks and selectively distributed processing. Rev Neurol (Paris) 1994; 150: 564-9. 
79. Mesulam MM. From sensation to cognition. Brain 1998; 121 ( Pt 6): 1013-52.

80. Moritz-Gasser S, Herbet G, Duffau H. Mapping the connectivity underlying multimodal semantic processing in humans. Neuropsychologia in press.

81. Mummery CJ, Patterson K, Hodges JR, Wise RJ. Generating 'tiger' as an animal name or a word beginning with T: differences in brain activation. Proc Biol Sci 1996; 263: 98995.

82. Ojemann G, Mateer C. Human language cortex: localization of memory, syntax, and sequential motor-phoneme identification systems. Science 1979; 205: 1401-3.

83. Pallud J, Mandonnet E, Duffau H, et al. Prognostic value of initial magnetic resonance imaging growth rates for World Health Organization grade II gliomas. Ann Neurol 2006; 60: 380-3.

84. Pallud J, Capelle L, Taillandier L, et al. Prognostic significance of imaging contrast enhancement for WHO grade II gliomas. Neuro Oncol 2009; 11: 176-82.

85. Pallud J, Duffau H, Razak RA, et al. Influence of pregnancy in the behavior of diffuse gliomas: clinical cases of a French glioma study group. J Neurol 2009; 256: 2014-20.

86. Pallud J, Fontaine D, Duffau H, et al. Natural history of incidental World Health Organization grade II gliomas. Ann Neurol 2010; 68: 727-33.

87. Pallud J, Mandonnet E, Deroulers C, et al. Pregnancy increases the growth rates of World Health Organization grade II gliomas. Ann Neurol 2010; 67: 398-404.

88. Pallud J, Varlet P, Devaux B, et al. Diffuse low-grade oligodendrogliomas extend beyond MRI-defined abnormalities. Neurology 2010; 74: 1724-31.

89. Pallud J, Blonski M, Mandonnet E, et al. Velocity of tumor spontaneous expansion predicts long-term outcomes for diffuse low-grade gliomas. Neuro Oncol 2013; 15: 595-606.

90. Parker GJ, Luzzi S, Alexander DC, et al. Lateralization of ventral and dorsal auditorylanguage pathways in the human brain. Neuroimage 2005; 24: 656-66.

91. Peyre M, Cartalat-Carel S, Meyronet D, et al. Prolonged response without prolonged chemotherapy: a lesson from PCV chemotherapy in low-grade gliomas. Neuro Oncol 2010; 12: 1078-82.

92. Pignatti F, van den Bent M, Curran D, et al. Prognostic factors for survival in adult patients with cerebral low-grade glioma. J Clin Oncol 2002; 20: 2076-84.

93. Postma TJ, Klein M, Verstappen CC, et al. Radiotherapy-induced cerebral abnormalities in patients with low-grade glioma. Neurology 2002; 59: 121-3. 
94. Rauschecker JP, Scott SK. Maps and streams in the auditory cortex: nonhuman primates illuminate human speech processing. Nat Neurosci 2009; 12: 718-24.

95. Ricard D, Kaloshi G, Amiel-Benouaich A, et al. Dynamic history of low-grade gliomas before and after temozolomide treatment. Ann Neurol 2007; 61: 484-90.

96. Rorden C, Karnath HO, Bonilha L. Improving lesion-symptom mapping. J Cogn Neurosci 2007; 19: 1081-8.

97. Sanai N, Berger MS. Glioma extent of resection and its impact on patient outcome. Neurosurgery 2008; 62: 753-64; discussion 264-6.

98. Sanai N, Mirzadeh Z, Berger MS. Functional outcome after language mapping for glioma resection. N Engl J Med 2008; 358: 18-27.

99. Sanai N, Chang S, Berger MS. Low-grade gliomas in adults. J Neurosurg 2011; 115: 948-65.

100. Sarubbo S, De Benedictis A, Maldonado IL, et al. Frontal terminations for the inferior fronto-occipital fascicle: anatomical dissection, DTI study and functional considerations on a multi-component bundle. Brain Struct Funct 2013; 218: 21-37.

101. Saur D, Kreher BW, Schnell S, et al. Ventral and dorsal pathways for language. Proc Natl Acad Sci U S A 2008; 105: 18035-40.

102. Schwartz MF, Faseyitan O, Kim J, Coslett HB. The dorsal stream contribution to phonological retrieval in object naming. Brain 2012; 135: 3799-814.

103. Schwartz S, Baldo J, Graves RE, Brugger P. Pervasive influence of semantics in letter and category fluency: a multidimensional approach. Brain Lang 2003; 87: 400-11.

104. Shaw E, Arusell R, Scheithauer B, et al. Prospective randomized trial of low- versus high-dose radiation therapy in adults with supratentorial low-grade glioma: initial report of a North Central Cancer Treatment Group/Radiation Therapy Oncology Group/Eastern Cooperative Oncology Group study. J Clin Oncol 2002; 20: 2267-76.

105. Shaw EG, Wang M, Coons SW, et al. Randomized trial of radiation therapy plus procarbazine, lomustine, and vincristine chemotherapy for supratentorial adult lowgrade glioma: initial results of RTOG 9802. J Clin Oncol 2012; 30: 3065-70.

106. Smith JS, Chang EF, Lamborn KR, et al. Role of extent of resection in the long-term outcome of low-grade hemispheric gliomas. J Clin Oncol 2008; 26: 1338-45.

107. Smits A, Duffau H. Seizures and the natural history of World Health Organization Grade II gliomas: a review. Neurosurgery 2011; 68: 1326-33.

108. Soffietti R, Baumert BG, Bello L, et al. Guidelines on management of low-grade gliomas: report of an EFNS-EANO Task Force. Eur J Neurol 2010; 17: 1124-33. 
109. Spena G, Garbossa D, Barletta L, et al. Preoperative chemotherapy for infiltrative lowgrade oligoastrocytoma: a useful strategy to maximize surgical resection -case report. Neurol Med Chir (Tokyo) 2010; 50: 410-3.

110. Stupp R, Mason WP, van den Bent MJ, et al. Radiotherapy plus concomitant and adjuvant temozolomide for glioblastoma. N Engl J Med 2005; 352: 987-96.

111. Surma-aho O, Niemela M, Vilkki J, et al. Adverse long-term effects of brain radiotherapy in adult low-grade glioma patients. Neurology 2001; 56: 1285-90.

112. Taillandier L, Duffau H. Epilepsy and insular Grade II gliomas: an interdisciplinary point of view from a retrospective monocentric series of 46 cases. Neurosurg Focus 2009; 27: E8.

113. Taphoorn MJ, Klein M. Cognitive deficits in adult patients with brain tumours. Lancet Neurol 2004; 3: 159-68.

114. Teixidor P, Gatignol P, Leroy M, et al. Assessment of verbal working memory before and after surgery for low-grade glioma. J Neurooncol 2007; 81: 305-13.

115. Tricot J, Aristote. La Politique. Paris: Vrin, 1962.

116. Tulving E. Multiple memory systems and consciousness. Hum Neurobiol 1987; 6: 6780.

117. Ture U, Yasargil MG, Friedman AH, Al-Mefty O. Fiber dissection technique: lateral aspect of the brain. Neurosurgery 2000; 47: 417-26; discussion 26-7.

118. Ueno T, Saito S, Rogers TT, Lambon Ralph MA. Lichtheim 2: synthesizing aphasia and the neural basis of language in a neurocomputational model of the dual dorsal-ventral language pathways. Neuron 2011; 72: 385-96.

119. van den Bent MJ, Afra D, de Witte O, et al. Long-term efficacy of early versus delayed radiotherapy for low-grade astrocytoma and oligodendroglioma in adults: the EORTC 22845 randomised trial. Lancet 2005; 366: 985-90.

120. Vigneau M, Beaucousin V, Herve PY, et al. Meta-analyzing left hemisphere language areas: phonology, semantics, and sentence processing. Neuroimage 2006; 30: 141432.

121. Wernicke C. The aphasic symptom-complex. A psychological study on an anatomical basis. Archives of neurology 1874; 22: 280-82.

122. Yamada K, Nagakane Y, Mizuno T, et al. MR tractography depicting damage to the arcuate fasciculus in a patient with conduction aphasia. Neurology 2007; 68: 789. 
123. Yordanova YN, Moritz-Gasser S, Duffau H. Awake surgery for WHO Grade II gliomas within "noneloquent" areas in the left dominant hemisphere: toward a "supratotal" resection. Clinical article. J Neurosurg 2011; 115: 232-9.

124. Zola-Morgan S. Localization of brain function: the legacy of Franz Joseph Gall (17581828). Annu Rev Neurosci 1995; 18: 359-83.

125. Zouaoui S, Rigau V, Mathieu-Daude H, et al. [French brain tumor database: general results on 40,000 cases, main current applications and future prospects]. Neurochirurgie 2012; 58: 4-13. 


\section{Figures et tableaux}

Figure A. Courbes de survie des malades en fonction de la stratégie thérapeutique adoptée............p7

Figure B. Courbes de survie des malades en fonction de l'étendue de l'exérèse chirurgicale..........p9

Figure C. Résultats de la chirurgie des gliomes sans et avec SED ..........................................p11

Figure D. Evolution du diamètre tumoral moyen (DTM) avant, pendant, et après

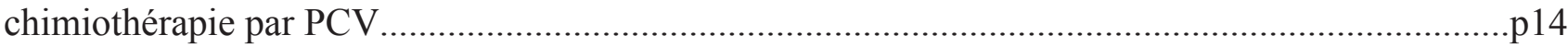

Figure E. Le modèle général de traitement parallèle et distribué (d'après Mc Clelland 1994)........p17

Figure F. Paul Broca et le centre de la parole..................................................................................p18

Figure G. Carl Wernicke (1848-1905) et sa représentation du réseau du langage

(tiré de sa thèse de médecine, 1874) ........................................................................................p19

Figure H. Ludwig Lichtheim (1845-1928) et sa représentation du réseau du langage paru

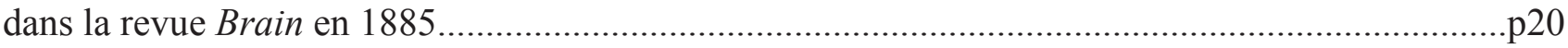

Figure I. Norman Geschwind (1926-1984) et sa représentation du réseau du langage publié dans la revue Science en 1970

Figure J. Modèle hodotopique du langage, établi à partir de corrélations anatomo-fonctionnelles per-opératoires par SED.

Figure 1. Lesion overlap map of the 31 patients in the left hemisphere............................p27

Figure 2. Histograms representing verbal fluencies Z-scores at the 3 periods of evaluation...........p32

Figure 3. Preoperative statistical voxel-based lesion-behavior mapping (VLBM) analyses

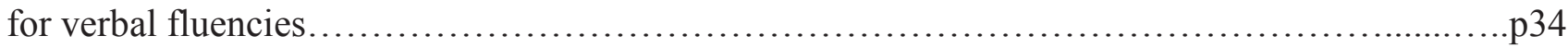

Figure 4. Overlap of ventral and dorsal stream fascicles with the preoperative VLBM map for semantic fluency and the Spearman correlation scatterplots for semantic Z-scores and the two fascicles infiltrated voxels from the Catani DTI atlas.... p36

Figure 5. Postoperative cavity voxel-based lesion-behavior mapping (VLBM) analyses for verbal fluencies, at early postoperative period and 3 months after surgery.... .p37

Tableau 1 : critères histologiques de classification des gliomes (OMS) ...................................p4

Table 1. Demographic data and verbal fluency Z-scores of all patients....................................p29 


\section{Serment d'Hippocrate}

$\mathcal{A} u$ moment dêtre admis à exercer la médecine, en présence de mes Maîtres et de mes chers condisciples et devant leffigie dHippocrate, je promets et je jure dêtre fídèle aux lois de Chonneur et de la probité dans lexercice de la Médecine.

Je donnerai mes soins gratuits à lindigent et à quiconque me les demandera. Je n'exigerai jamais un salaire au-dessus de mon travail.

J'informerai les patients des décísions envisagées, de leurs raisons et de leurs conséquences.

Admis dans lintimité des personnes, je tairai les secrets qui me seront confiés. Reçu à Cintérieur des maisons, je respecterai les secrets des foyers et ma conduite ne servira pas à corrompre les mours.

Je ferai tout pour soulager les souffrances. Je ne prolongerai pas abusivement les agonies. Je ne provoquerai jamaís la mort délibérément.

Je n'entreprendrai rien qui dépasse mes compétences. Je les entretiendrai et les perfectionnerai pour assurer au mieux les services qui me seront demandés.

Respectueux et reconnaissant envers mes Maîtres, je rendraí à leurs enfants Cinstruction que j’ai reçue de leur père.

Que les hommes et mes confrères m'accordent leur estíme si je suís fídèle à mes promesses; que je sois déshonoré et méprisé sí j’y manque. 